\title{
U⿱宀⿻三丨口
}

\section{A single predator multiple prey model with prey mutation}

Mullan, R., Abernethy, G. M., Glass, D. H., \& McCartney, M. (2016). A single predator multiple prey model with prey mutation. Communications in Nonlinear Science and Numerical Simulation, 40, 51-70.

https://doi.org/10.1016/j.cnsns.2016.04.013

Link to publication record in Ulster University Research Portal

\section{Published in:}

Communications in Nonlinear Science and Numerical Simulation

\section{Publication Status:}

Published (in print/issue): 30/11/2016

DOI:

10.1016/j.cnsns.2016.04.013

\section{Document Version}

Author Accepted version

\section{General rights}

Copyright for the publications made accessible via Ulster University's Research Portal is retained by the author(s) and / or other copyright owners and it is a condition of accessing these publications that users recognise and abide by the legal requirements associated with these rights.

\section{Take down policy}

The Research Portal is Ulster University's institutional repository that provides access to Ulster's research outputs. Every effort has been made to ensure that content in the Research Portal does not infringe any person's rights, or applicable UK laws. If you discover content in the Research Portal that you believe breaches copyright or violates any law, please contact pure-support@ulster.ac.uk. 


\title{
A Single Predator Multiple Prey Model with Prey Mutation
}

\author{
Rory Mullan, Gavin M. Abernethy, David H. Glass and Mark McCartney
}

School of Computing and Mathematics, University of Ulster, Jordanstown campus, Shore Road, Newtownabbey, Co. Antrim, BT37 0QB, UK

\begin{abstract}
A multiple species predator-prey model is expanded with the introduction of a coupled map lattice for the prey, allowing the prey to mutate discretely into other prey species. The model is examined in its single predator, multiple mutating prey form. Two unimodal maps are used for the underlying dynamics of the prey species, with different predation strategies being used. Conclusions are drawn on how varying the control parameters of the model governs the overall behaviour and survival of the species. It is observed that in such a complex system, with multiple mutating prey, a large range of nonlinear dynamics is possible.
\end{abstract}

\section{Introduction}

The simplest form of mathematical model for predator-prey dynamics is a two species model, where a single predator predates upon a single prey. While in principle such models may be generalised to multiple species systems, where both multiple predators and multiple prey occupy the ecosystem, in practice such systems are often not analytically tractable, and can require significant resources to simulate computationally. Ecosystems can be modelled using a range of techniques from discrete and continuous time models from the level of populations to individual agent based simulations [1,2].

In the field of ecological modelling, a lot of recent research has focused on two species predator-prey models, which have been used to investigate chaotic population dynamics [37], the effect of the prey growth rate [8] and population dispersal [9-11]. In comparison, less work has been undertaken looking at multiple species predator-prey models where the system has been expanded to allow for multiple competing predators and prey. Work in this area has been undertaken examining the dynamic behaviour and chaotic orbits that can occur in these models [12,13], and the effect of implementing various functional responses (the effect of predation, per predator, upon the prey species) upon the dynamics $[14,15]$.

The modelling of a functional response is one of the most studied aspects of mathematical ecology, with Holling's Type II disc equation [16] proving particularly popular. Several sophisticated forms have been proposed, and the question of which of these is the most

\footnotetext{
*Email: mullan-r8@email.ulster.ac.uk, abernethy-g1@email.ulster.ac.uk,dh.glass@ulster.ac.uk, m.mccartney@ulster.ac.uk
} 
biologically-justified has been the subject of recent debate [17]. Other studies have suggested that models featuring nonlinear functional responses and adaptive foraging may be essential for the maintenance of stable, complex ecosystems [18]. This is the dominant question of this subject [19] - the relationship between complexity and stability that has overshadowed theoretical ecology since May’s 1973 [20] work on random graphs challenged the intuitive belief that greater complexity increases the stability of an ecosystem. This question has been explored in some detail using both models and empirical data, examining the effect upon numerous concepts of "stability" [21] under variation of properties of food webs such as predator-prey ratios, the proportion of possible feeding links in a system that are realised [22], the effects of competition between species, and the proportion of 'weak' feeding links [23].

A key area of the study presented in this paper is the inclusion of mutation in a predator-prey ecosystem. Mutation has previously been introduced into both single species predator-prey ecosystems [24,25] where it was used to simulate adaption towards the environment, and in multiple species predator-prey models [26] where the various traits of the predator and prey are allowed to evolve, introducing new species into the ecosystem. A recent approach building on the static food web models found in the ecological literature in the 1990s has been the development of several eco-evolutionary models that seek to combine classical population dynamics with the creation of new species by random mutation, and the maintenance of the ecosystem by natural selection [27-32]. A key feature of these models is that the species themselves are not pre-selected, and the trophic relationships that are present in the resulting food web are an emergent result of the selection process operating on population dynamics. An overview of the development of one such model in the light of historical food web research can be found in [33].

A similar area of study relates to the modelling of predator-prey meta-populations, where a single predator and single prey species occupy a spatial plane, with the two species able to move around and interact on the plane. This creates a predator-prey model that models the dynamics of the species in both space and time. There are several ways of modelling this: reaction-diffusion based predator-prey models [34,35], kernel based models [9], and coupled map lattice (CML) based models [36-38]. The most relevant of these to the study presented here are the CML-based implementations, which model the predator and prey as discretelycoupled elements on a lattice. Studies in the physics literature have considered the dynamics 
on such structures [39-40], and they have been applied to modelling population dynamics on spatial systems of plants [41] and insects [42]. Multiple-species predator-prey relationships on a two-dimensional lattice were studied using the discrete generalised Lotka-Volterra equations in [43]. This approach has the advantage of being relatively simple to compute, whilst introducing a natural ordering which allows the constraint that species may only move to an adjacent region on the lattice. By varying the species' intrinsic growth rates along the lattice, we may model differences in sustaining life between the environments. How these ideas are implemented in the present study will be discussed in more detail later.

This paper uses a generalised multiple species form of a discrete time predator-prey model proposed by Neubert and Kot [3]. Predation strategies, which dictate how the predators go about hunting the prey, have been included in a previous generalisation of the Neubert and Kot model which has been studied by Mullan et al [44]. The model here is expanded with the introduction of a CML to govern the dynamics of the prey, which has several advantages as noted above. This allows the prey to mutate discretely into other prey species and is executed with a single predator hunting upon the set of mutating prey. Building upon previous work in this way results in a multiple species model that is computationally feasible and capable of yielding insights on the range of dynamics occurring in such a system, the role of mutation and the important issue of ecosystem stability. After describing the model, results will then be presented, first showing a broad overview of where survival occurs in the model based on the assigned control parameters, and then with a focus being placed on the underlying dynamic behaviour of the species within the model as its configuration changes. Both the Ricker and logistic maps are used to model the prey dynamics with comparisons being drawn between these two unimodal maps.

\section{Multiple species predator-prey model}

The following single species discrete time predator-prey model was proposed by Neubert and Kot [4]

$$
\begin{gathered}
N_{t+1}=N_{t} e^{r\left(1-N_{t}-P_{t}\right)} \\
P_{t+1}=c N_{t} P_{t}
\end{gathered}
$$


where $N_{t}$ represents the prey population at time step $t$ and $P_{t}$ represents the predator population at time step $t$. Two control parameters here define the behaviour of the predator and prey: $c$ defines the effectiveness of the predator at predating upon the prey and $r$ defines the reproduction rate of the prey. The Ricker model is being used to model the behaviour of the prey.

In previous work [44], this model was generalised as follows to allow for multiple predators and prey to occupy the ecosystem:

$$
\begin{gathered}
N_{t+1}^{(j)}=\exp \left(-\left(\sum_{i=1}^{m}\left(f^{(i j)} c_{i j}\right) P_{t}^{(i)}\right)\right) N_{t}^{(j)} \exp \left(r_{j}\left(1-N_{t}^{(j)}\right)\right) \\
P_{t+1}^{(i)}=\sum_{j=1}^{n}\left(f^{(i j)} c_{i j}\right) N_{t}^{(j)} P_{t}^{(i)}
\end{gathered}
$$

where $N_{t}^{(j)}$ represents the $\mathrm{j}^{\text {th }}$ prey species at time step $t$ and $P_{t}^{(i)}$ represents the $i^{\text {th }}$ predator species at time step $t$, with $c_{i j}$ and $r_{j}$ acting as the control parameters. Whereas (1b) can be generalised straightforwardly to (2b), (1a) is harder to generalise. A direct generalisation of (1a) does not distinguish between the differing effectiveness of predator $i$ in depleting the prey $j$ 's population as it contains no dependence on the control parameter $c$. It is therefore reasonable to include the term $c_{i j}$ as given in (2a).

The two generalised equations allow for $m$ predators and $n$ prey to occupy the ecosystem, with each prey having an individual $r$ value corresponding to its population growth rate, and a $c_{i j}$ term, which measures the predatorial effectiveness of the $i^{\text {th }}$ predator at predating upon the $j^{\text {th }}$ prey. The term $f^{(i j)}$ is introduced to model how predator $i$ divides its effort hunting the set of prey species. The inclusion of this term is necessary to permit co-survival of predator and prey species in the model as the number of species in the ecosystem increases. An extensive study of this model with the use of various predation strategies can be found in [43].

Here the model is further expanded with the introduction of mutation amongst the prey. This is effectively a CML model forming the prey dynamics in the predator-prey ecosystem. The result for a single predator is to replace equation (2a) with:

$$
N_{t+1}^{(j)}=\sum_{k=1}^{n} p_{k j} \exp \left(-\left(\left(f^{(k)} c_{k}\right) P_{t}\right)\right) N_{t}^{(k)} \exp \left(r_{k}\left(1-N_{t}^{(k)}\right)\right)
$$


with a summation over the variable $k$ being introduced which runs over the $n$ prey species. Here the probability of prey $k$ mutating into prey $j$ is defined as $p_{k j}$.

Equation (3) uses the Ricker model to model the growth rate of the prey. As well as this, a further unimodal discrete time map has been used to govern the prey dynamics in the model. A predator-prey model where the logistic map has been used in place of the Ricker model is defined as:

$$
N_{t+1}^{(j)}=\sum_{k=1}^{n} p_{k j}\left(N_{t}^{(\mathrm{k})}+\left(r_{k} N_{t}^{(\mathrm{k})}\left(1-N_{t}^{(\mathrm{k})}\right)\right)-\left(N_{t}^{(\mathrm{k})}\left(f^{(k)} c_{k}\right) P_{t}\right)\right)
$$

where the terms are to be understood as for (3).

In this paper we use a variant of equation (2b) which for a single predator can be expressed as:

$$
P_{t+1}=P_{t} \sum_{j=1}^{n} f^{(j)} \max \left(0, c_{j} N_{t}^{(j)}-P_{t}\right)
$$

The assumption being made is that the predator population effectively has a carrying capacity on each prey. The reason for this choice is discussed further at the end of the next section.

The two mutation models described by (3,5) (for a Ricker map based system) and (4,5) (for a logistic map based system) allow for a single predator with a predation strategy defined by the array $\left[f^{j}\right]$ and predatorial effectiveness defined by the array $\left[c_{j}\right]$ to predate on $n$ mutating prey, with each prey having an individual value $r_{j}$ determining the growth rate of its population in absence of predation.

\subsection{Mutation}

The sum of the probabilities for each possible mutation of prey species $k$ to any of the other species $j, p_{k j}$, plus the probability that it does not mutate, $p_{k k}$, must sum to one,

$$
\sum_{j=1}^{n} p_{k j}=1 \quad \forall \mathrm{k} .
$$

Further, all the probabilities must lie in the range [0,1], i.e. 


$$
p_{k j} \in[0,1] \forall \mathrm{j}, \mathrm{k} .
$$

Nearest neighbour (N-N) mutation is employed to simulate mutation of the prey. Here the matrix takes the form:

$$
p_{k j}=\left\{\begin{array}{cc}
p & |j-k|=1 \\
1-2 p & j=k \neq 1 \text { or } \mathrm{n} \\
1-p & j=k=1 \text { or } \mathrm{n} \\
0 & \text { otherwise }
\end{array}\right.
$$

where $p$ is the probability of a prey mutating into a neighbouring species, $n$ being the total number of prey and the term 1- $p$ accounting for the prey species at the edges of the system. The fact that $p_{k j} \geq 0$ means the maximum probability of a prey mutating into a neighbouring prey is 0.5 in line with (6) above. Here each species will contribute half of its population to each of its neighbouring species in the next iteration of the model. This means that those populations that are not along the edges make no contributions to their own population at the next time step.

High mutation rates $\left(p_{k j}>0.1\right)$ would not be considered biologically relevant, since biological mutation occurs with much lower probabilities. However, if we were considering the model spatially, i.e. that $p_{k j}$ represented not the probability of mutation, but of migration to a neighbouring population group, then higher values of $p_{k j}$ would be reasonable, and so allowing for this interpretation, they are considered here.

\subsection{Assignment of control parameters}

The control parameter $r_{j}$ defines the growth rate of the prey species $j$ in the model. In the current work these have been scaled linearly across the prey species via

$$
r_{j}=\frac{j}{n} r_{\max }
$$

where $j \in\{1, \ldots, n\}$. This gives a linear distribution of the $r$ control parameter values from $r_{\max } / n$ to $r_{\max }$. It is this differing $r$ value for each prey's growth rate that draws a distinction between each of the prey species. 
In all cases $c_{j}=c$ meaning that the predator has an equal efficiency of predating upon all the prey species. A predation strategy which is dependent on the relative size of the prey populations is defined via:

$$
f^{(i)}=\frac{\left(N_{t}^{(i)}\right)^{\alpha}}{\sum_{j=1}\left(N_{t}^{(\mathrm{j})}\right)^{\alpha}}
$$

where $\alpha$ specifies an exponent with which the focus of the predator changes.

Six different values of $\alpha$ have been considered. $\alpha=0$, which corresponds to the predator focusing an equal amount of effort hunting each of the surviving prey species, regardless of their current population density, $\alpha=1$, where the predator distributes its effort towards the current population density of the prey species, modelling an opportunistic predator that attacks any prey that it encounters. Then with the increasing values of $\alpha$ of $\alpha=2, \alpha=4$ and $\alpha$ $=7$, with each increase of $\alpha$ increasing the predator's focus towards the prey species with the highest current population densities. Finally $\alpha=\infty$, is an extreme focus in which the predator exerts $100 \%$ of its effort predating upon the single prey, which has the highest current population density.

It is possible to interpret this model in terms of predator migration similar to other work in the field if we think of the model as a single predator species that utilises different predation strategies across a one dimensional space. Instead of interpreting the prey as different species, we can instead interpret each prey to be a different area, with a different sub-population of a prey species. With this interpretation $\alpha$ dictates how much time the predator is spending predating in each area. At $\alpha=0$, the predator is spending an equal amount of time in each area, no matter how densely populated the areas are with prey, and then as $\alpha$ grows the predator is starting to divert its predation effort towards areas which contain a greater density of prey. The $\alpha=\infty$ case can then be interpreted as the entire pack of the predator species migrating together, and solely focusing on the area in which the greatest density of the prey exist. The differing $r$ values could be interpreted as the different areas having different characteristics that affect the growth rate of the prey.

Finally, we return to the choice of predator dynamics given by equation (5). This choice is made to prevent a pathological scenario which could otherwise arise is cases where the 
predator uses a focusing strategy as described by (10). Namely, a predator can deplete one prey population, increase its population, then in the next time-step move to another prey (which is now the largest), again increase its population, and so on. At each time step the prey populations which are not being predated on have a chance to recover, to be predated on at a later time step. In some scenarios this allows the predator population to increase without bound. The use of the carrying capacity term in (5) prevents this.

\section{Numerical Results}

The two predator-prey models outlined in (3), (4) and (5) above have been run for a 10 prey, single predator ecosystem. In the logistic model the maximum value for which the prey species will survive is $r=3$, and so $r_{\max }$ has therefore been fixed to $r_{\max }=3$ for all runs in both the logistic and Ricker based models. This allows for direct comparison to be drawn between the two unimodal discrete time maps.

For all runs $N_{1}$ is populated with an initial population density of $N_{1}=0.5$. The system is then run for 1000 time steps after which the predator is introduced with a population density of $P_{0}$ $=0.5$. This means that at the time of introduction of the predator, all prey species are populated, and behaving as they would in their CML form with no predation. After the introduction of a predator the predator-prey ecosystem is then run for 9,000 time steps before results are collected over the next 1,000 time steps. 10,000 time steps were deemed to be sufficient for the system to converge upon its post transient state. It was found that running for a greater number of iterations did not show significant differences in the output of the model. Populations which fell below $10^{-16}$ were set to zero.

\subsection{Area of Survival}

Figures 1 and 2 show species survival as predation rate $c$ and mutation rate $p$ vary for the logistic and Ricker based models respectively for a range of predation strategies defined by $\alpha$. Figure 1 presents results for both the predator and prey for the logistic based model. . In Figure 1(a) where $\alpha=0$ the predator population survives between $c=1$ and $c=4.7$, with the boundary of predator survival becoming fragmented as $c$ approaches 5 and the mutation rate $p$ is varied. Between $c=3$ and $c=4.7$ the predator survival is highly reliant on mutation rate $p$. For some values of $p$ and $c$ the predator survives an initial decline due to overhunting. Thus scenarios occur where within the first 5 iterations the predator overhunts the prey, causing a decline in its own population, followed by a recovery of both the prey and predator 
populations and the post transient stabilisation of the ecosystem. In the cases where the predator dies off it overhunts the prey to the point where it cannot support its own population at all and so it quickly dies off. The prey here are surviving between $c=0$ and $c=8.6$, with the area of survival again being fragmented above $c=5.5$. This is due to the predator either overhunting the prey populations to complete extinction, or leaving extremely small prey populations at the time of predator collapse, which eventually allows survival of all prey populations as mutation causes repopulation, with the prey populations reverting back to their CML form. Above $c=8.6$ the predator hunts the prey to extinction in all cases. There are five distinct regions of predator and prey survival displayed here. With $c<1$ a region exists where the predator's $c$ value is too low to support itself, it quickly dies off leaving the prey to survive while never having much impact on the prey in the transient phase. This region is present for all values of $\alpha$ and in both the Ricker and logistic based models and will not be discussed further. There is also a stable co-survival region between $c=1$ and $c=3$, where co-survival of the predator and prey species occurs for all values of $p$, a fragmented region of predator survival between $c=3$ and $c=4.7$ discussed above, a fragmented region of prey survival without predator survival seen between $c=4.7$ and $c=8.6$ and the region of complete extinction above $c=8.7$. It is of note here that mutation has had little impact on regions of survival.

In 1(b) the predator population survives between $c=1$ and $c=3.83$, here the fractured edges on predator survival have become much less pronounced, occurring between $c=3.5$ and $c=$ 3.83. The region discussed in Figure 1(a), where the predator kills off all the prey populations for all values of $p$ at high values of $c$, no longer exists. Two things are of note when comparing the behaviour in Figure 1(a) and 1(b). The space in which co-survival occurs has declined in size in comparison to Figure 1(a) along the $c$ axis, and the area in which the prey survive in the absence of a predator has increased. This is a trend that continues as the value of $\alpha$ increases further: in Figure 1(c) co-survival occurs between $c=1$ and $c=3.5$, in Figure 1(d) the predator population survives between $c=1$ and $c=2.81$ and in in Figure 1(e) the predator population survives between $c=1$ and $c=2.4$. This shows that as $\alpha$ increases the space in which co-survival occurs has declined in size. The area in which the prey species survive in the absence of the predator however is increasing; with it becoming less fractured between areas of prey extinction and survival as $\alpha$ grows. When $\alpha=4$ and $\alpha=7$ the prey are successfully surviving for the vast majority of $(c, p)$ space. It should be noted that the mutation rate $p$ has very little effect on the area of survival in all these cases. 
In 1(f), where $\alpha=\infty$, co-survival occurs between $c=1$ and $c=3.4$. This is an increase in area in comparison to $1 €$ where $\alpha=7$ but smaller than the $\alpha \leq 2$ cases. The predator here is never hunting the prey species to extinction, all prey species survive for all values of $c$ and $p$. The death of the predator is caused by the predator population growing too large to be supported by the prey species, with the predator therefore becoming extinct due to the use of (5). This strategy behaves differently to the trend where increasing the value of $\alpha$ is decreasing the maximum $c$ value at which co-survival is seen. This is because when $\alpha=\infty$ the strategy becomes a single predator predating upon a single prey within a CML at each iteration, and from (5) there is an increased risk that over predation on this single species will lead to predator collapse.

The simplest strategies here allow co-survival for the greatest region of initial conditions. The greatest co-survival area is seen when $\alpha=0$, which models a species which divides its hunting effort up amongst all surviving species evenly. The region then decreases when $\alpha=$ 1 , which models an opportunistic predator which attacks any prey which it encounters, and then decreases further still as $\alpha$ grows, modelling predators which spend increasingly more effort hunting prey species with the highest current population density.

Figure 2 shows survival results obtained using the Ricker based predator-prey model. The main difference compared to the logistic model is that for all the values of $\alpha$ considered, every prey species now survives for all parameter choices, including the regions of large $c$ where they were driven to extinction when using the logistic model. For this reason, Figure 2 only shows results for predator survival. The Ricker model clearly has significant benefits for prey survivability, and this is independent of the presence of predators and the strength of predation.

For all values of $\alpha$, a clear structure is visible of a column in $c$ where the predator may survive for most or all values of $p$. The lower boundary in $c$ necessary to sustain the predator is almost independent of $p$ in all cases, and the upper boundary starts with a very fractured structure in $p$ for lower values of $\alpha$, but becomes smoother and less $p$-dependent as the predator becomes more focused. In particular, rate of mutation has relatively little effect on predator survival when $\alpha$ is greater than 1 . This column of survival is similar to that observed when using the logistic model for the prey, but there are some differences. The upper boundary in $c$ is much smoother than the corresponding boundary for a given value of $\alpha$ when 
using the logistic model, and the column is slightly narrower in the Ricker case - that is, there are some values of $c$ that will permit predator survival in the logistic case but not the Ricker case. The maximum value of $c$ that allows predator survival decreases as $\alpha$ increases, reducing from approximately $c=5.6$ when $\alpha=0$ to $c=1.8$ for $\alpha=\infty$, which contrasts with values of $c=8.7$ and $c=3.4$ respectively when using the logistic model.

Another interesting feature of this column structure, which only occurs for the Ricker model, and which persists for all values of $\alpha$ considered, is the region of low $p$ where this region of survival becomes noticeably wider. In section 3.3 we will discuss the dynamic properties of the system that this region corresponds to. The minimum value of $c$ necessary to facilitate predator survival is approximately 1 for the case $\alpha=0$, and decreases slightly as $\alpha$ is increased in contrast to the logistic model where it remains closer to $c=1$ for all values of $\alpha$. That is, as the predator becomes more focused, it is able to survive reduced rates of effectiveness when the Ricker model is used. In general, however, the predator survival plots for $\alpha=2,4,7, \infty$ are similar and, in particular, there is much less difference between the plots for $\alpha=7$ and $\alpha=\infty$ when using the Ricker model compared to the logistic model.

Overall, despite some differences in predator survival between the Ricker and logistic models, both exhibit similar behaviour. In particular, in both models the predator becomes extinct when its predatorial effectiveness $c$ is too small, predator survival is largely independent of $\mathrm{p}$ for $\alpha>1$, and predators survive for a range of values of $c$, but die out once $c$ becomes too high.

\subsection{Bifurcation diagrams for the logistic based model}

Figure 3 presents bifurcation diagrams showing predator behaviour in the logistic based predator-prey model with different values of $\alpha$. The range of $c$ values between $c=0$ and $c=5$ in increments of 0.5 are presented with the $p$ parameter being varied. The predator's dynamics can display a broad range of behaviour as the $p$ parameter changes. This includes the predator population stabilising on fixed values, bifurcating, period bubbling and chaotic behaviour.

Figure 3(a) presents a bifurcation diagram showing the predator's behaviour where $\alpha=0$. This is the predation strategy which allows predator survival for the widest range of $c$, as 
noted when discussing Figure 1. The conditions under which the predator survives in this plot fall between $c=1.5$ and $c=4.5$. For the lowest values of $c$, where co-survival occurs $(c=1.5)$ the system's dynamics largely appear to be in a chaotic state for the full range of $p$, with some narrow windows of periodic behaviour within the chaos. As $c$ grows to $c=2$, the system's dynamics generally behave chaotically with $p<0.415$, above which the ecosystem's dynamics period half out of a chaos, settling on a period 5 orbit, and no longer displaying chaotic dynamics when $p>0.415$. This stabilising behaviour is seen for higher values of $c$ : at $c=2.5$ the dynamics period half out of the chaotic state at $p=0.228$ settling on a period two orbit with $p>0.47$, at $c=3$ the system period halves out of chaos at $p=0.09$ with the output falling upon a period two orbit for $p>0.24$.

When $c>3$, the survival and behaviour of the predator species is erratic, this lies within the fragmented boundary of predator survival for high $c$ values noted in the discussion of Figure 1(a). Here the predator either collapses or enters a chaotic orbit as the mutation rate $p$ varies. With $c=3.5$, the dynamics are periodic for all values of $p>0.04$, while with $c>3.5$, the dynamics become erratic in the co-survival area, with the ecosystem behaving chaotically in the event of the predator surviving. When discussing Figure 1 it was noted that the space in which the co-survival occurs shows little reliance on the mutation rate $p$, however Figure 3 show that the underlying dynamics of the ecosystem are highly dependent upon the mutation rate.

The effect of $c$ on the population density of the predator can also be seen in Figure 3(a). Increasing the value of $c$ increases the predator's maximum population density up to the point where the predator becomes overly effective and therefore collapses. At $c=1.5$, the predator's population dynamics fall between $\mathrm{P}=0.3$ and $\mathrm{P}=0.44$. As $c$ increases to $c=2$, the dynamics of the predator population now fall between $\mathrm{P}=0.55$ and $\mathrm{P}=0.8$. This trend continues until $c=4.5$, where the predator dynamics now fall between $\mathrm{P}=0.46$ and $\mathrm{P}=1.98$. As the predator gets more effective at predating on the prey, it is able to support a higher population as is visible across the full range of $\alpha$ values in Figure 3. This is reflected in the prey's behaviour shown in Figure 4, which shows the corresponding bifurcation diagrams for the $N_{1}$ prey population for each value of $\alpha$. This prey species has an $r=0.3$ control parameter due to (9). In Figure 4, where the prey survives in the absence of predation, the prey species will revert back to its predation free CML. If it survives in the absence of a predator, this prey species will converge upon $N_{l}=1$. In Figure 4(a), with $c=1.5$, the $N_{l}$ population is being 
reduced so that its behaviour is falling within the range, $N_{1}=0.81$ to $N_{1}=0.9$. When $c=2$, the $N_{l}$ population has been further reduced so that the output now lies within the range $N_{l}=$ 0.55 to $N_{l}=0.7$. This trend continues further as $c$ increases, the $N_{l}$ prey population is being reduced as the predator becomes more effective at predating upon it. This is in line with the observation that the predator population density is increasing as $c$ increases. Figure 5 shows this with predator and $N_{l}$ with an increasing value of $c$ and $\alpha=0$. It can be seen that the average rate of predator survival is increasing as $c$ increases, which in turn is causing a decline in the average rate of the $N_{l}$ prey population. Upon checking the rest of the prey species behaviour, it was confirmed that this trend is apparent across all the prey species. As $c$ increases, the predators have a greater impact across all prey species, decreasing their average population density.

As noted in the discussion of Figure 1, as the predation strategy defined by $\alpha$ increases the maximum $c$ for which co-survival occurs decreases. Due to the increment value of 0.5 on $c$ this is not always obvious when inspecting the plots in Figure 3. However, as a general trend, it is observable. In Figure $3 \mathrm{~b}$ where $\alpha=1$ the predator has survived between $c=1.5$ and $c=$ 3.5, in Figure 3(c) where $\alpha=2$ the predator has survived between $c=1$ and $c=3.5$. The predator here with $c=1$ has survived where it became extinct at the lower values of $\alpha$, but in both of these cases survival occurs at $c=3.5$. In Figure 3(d), however, where $\alpha=4$, the predator has only survived between $c=1$ and $c=2.5$ and this is also the case in Figure 3(e) where $\alpha=7$. In Figure 3(f) with $\alpha=\infty$ the predator has survived between $c=1$ and $c=3$, an increase as noted above when discussing Figure 1(f) due to the different behaviour when $\alpha=$ $\infty$.

Stabilising behaviour, where the system period halves out of chaos and remains on a periodic attractor for all remaining values of $p$, noted when discussing Figure 3(a), occurs in Figure 3(b) with $\alpha=1$ for $c=2.5,3$ and 3.5 for values of $p>0.46,0.4$ and 0.43 respectively. With $\alpha$ $>1$ this stabilising behaviour is no longer seen. With $\alpha=1$ and $\alpha=2$, and with $c=3.5$, the maximum plotted value for which co-survival survival is occurring, the ecosystem's dynamics are generally behaving chaotically, with the predator population covering a wide range of values. With $\alpha>4$, survival is no longer seen at $c=3.5$, with the maximum plotted value of co-survival being $c=2.5$ when $\alpha=4$ and $\alpha=7$. In figure 3(e) with $\alpha=7$, and $c=2.5$, co-survival only takes place between $p=0.31$ to $p=0.37$. The dynamics for the upper $c$ values for $\alpha=4$ and $\alpha=7$ display a visible reliance on the $p$ value, with long windows of 
periodic behaviour occurring in between chaotic dynamics. For $\alpha=4$, with $c=2.5$ a window occurs between $p=0.25-0.406$, where there are regions of periodic behaviour, and with $\alpha=$ 7 and $c=2$ a band occurs between $p=0.33-0.47$ where the behaviour is entirely periodic. Figure 6 provides examples of the intermittently chaotic orbits that can occur in the system.

Using the plots presented in Figure 3, several observations about the effect of $\alpha$ on the predator population density can be made. It can be seen that the range which the surviving predator's population is falling upon is increasing as $\alpha$ increases. If we look at Figure 3(a), when $\alpha=0$ and look at the $c=1.5$ plot, the range of the predator's populations is falling between $P=0.3$ and $P=0.44$. In Figure 3(b), with $\alpha=1$, the corresponding range lies between $\mathrm{P}=0.36$ and $\mathrm{P}=0.45$. This trend continues up to $\alpha=7$ in Figure 3(e), where the range now lies between $\mathrm{P}=0.46$ and $\mathrm{P}=0.75$. In Figure 3(f) with the extreme predation strategy of $\alpha$ $=\infty$ the behaviour is visibly different to the earlier $\alpha$ values, with the population much more erratic, and behaviour that suggests multiple attractors as $p$ changes. The corresponding population here is falling between $\mathrm{P}=0.56$ and $\mathrm{P}=0.77$. It is also of note that the more focused predation strategies $(\alpha \geq 2)$ are allowing predator survival at $c=1$.

In Figure 4 the interesting behaviour for the $N_{l}$ prey species lies within the region of cosurvival. Outside of this region it will either converge on $N_{l}=1$ (CML behaviour) or $N_{l}=0$ (hunted to extinction). Within this area of co-survival the $N_{l}$ prey population always stays below $N_{1}=1$ for all values of $c$ and for all values of $\alpha$. The behaviour of each plot can be directly correlated with the behaviour of the predator in the corresponding plot in Figure 3. When the predator is more successful, the prey's population in turn will be impacted to a greater degree. Since this is a coupled system between the predator and prey, the broad dynamics of the system are always linked; in any case where the predator population is chaotic all prey populations will also be chaotic, and where the predator population is periodic, all prey populations will also be periodic. The dynamics of the prey species therefore follow the underlying dynamics as discussed for Figure 3. A further observation is that the $N_{1}$ population is being impacted more by the predator at lower values of $p$ which will be commented on later.

The behaviour of the $N_{l}$ prey in Figure 4(f) is quite different from all the other cases. Here $\alpha$ $=\infty$ with the predator's strategy being to fully focus on the prey species with the highest current population density. The $N_{l}$ prey is the prey with the lowest $r$ value in the system, and is also an edge species. It only mutates into and receives contribution from a single 
neighbour. Interesting behaviour can be seen in the first prey species here in cases where the predator survives with a high $c$ value. If we observe the behaviour with $c=3$ and $p=0.25$, there are cases where in the bifurcation diagram it appears that the prey is on a periodic orbit. Investigation of the underlying dynamics here show that this prey species for the vast majority of its runtime is converged on $N_{l}=1$. When it becomes the most populous species of prey, the predator exerts all of its effort upon this prey, completely collapsing its population. It then receives mutation from its single neighbour, $\mathrm{N}_{2}$, which has a population density of $\mathrm{N}_{2}=1$ and therefore gives a $25 \%$ contribution and so the $N_{1}$ population becomes $N_{1}=0.25$. This process continues in the model, with slight growth caused by its small growth rate of $r=$ 0.3 and the neighbour's contribution it receives at each iteration, until the $N_{l}$ prey reconverges on $N_{l}=1$. When represented as a bifurcation diagram, this behaviour visually appears similar to a 'standard' periodic attractor, but in fact it is the population being killed off, and then gradually stepping back into its post transient state.

Figure 7 shows a plot of the average population density of the predator and each of the prey with the logistic based predator-prey model and $c=2$ against the $p$ control parameter for different values of the focus strength $\alpha$. Within the following discussion $\bar{P}$ is used to denote the average predator population. The model is run for 10,000 iterations for each value of $p$, with an average being taken over the last 1000 iterations of the 10,000 time step runtime. These graphs allow us to see the effect that the predation strategy has on the average survival rate of the predator and prey species. From the earlier observation made using Figure 3, it is apparent that as $\alpha$ increases the predator's population density in the model is also increasing.

In Figure 7(a) the average population density for the predator lies between $\bar{P}=0.65$ and $\bar{P}=$ 0.7 , with a peak average population of $\bar{P}=0.73$ at $p=0.1$ and the minimum average of $\bar{P}=$ 0.66 appearing at $p=0.01$. Above $p=0.22$ the average population of the predator becomes largely independent of the mutation rate, with $\bar{P}=0.68$ for all remaining $p$ values. In Figure 7(b) with $\alpha=1$, the average predator population density lies $\bar{P}=0.72$ to $\bar{P}=0.75$ with a peak of $\bar{P}=0.75$ occurring at $p=0.12$ and a minimum of $\bar{P}=0.72$ occurring at $p=0.5$. The mutation rate with this strategy holds more influence over the predator's average; above the peak at $p=0.12$, the predator's average population uniformly declines as $p$ increases, suggesting that high mutation of the prey with this strategy is detrimental to the predator's average survival. The average is higher for all values of $p$, however, in comparison to $\alpha=0$. In Figure 7(c) with $\alpha=2$, the average predator population density lies between $\bar{P}=0.76$ to $\bar{P}$ 
$=0.78$ with a peak of $\bar{P}=0.78$ occurring at $p=0.05$ and a minimum of $\bar{P}=0.76$ occurring at $p=0.5$. The behaviour is similar to the $\alpha=1$ case, but overall the impact of mutation is less. Again the minimum average population with this predation strategy is higher than the maximum average predator population density with the earlier predation strategies. This is a trend that continues as $\alpha$ increases. In Figure 7(d) with $\alpha=4$, the average predator population density lies between $\bar{P}=0.82$ to $\bar{P}=0.86$ with a peak of $\bar{P}=0.864$ occurring at $p=0.496$ and a minimum of $\bar{P}=0.0 .82$ occurring at $p=0.45$. The behaviour here is very similar to the previous cases for low values of $p$, with the average population growing to a relatively high value, and then gradually declining as $p$ grows, but here the average population sharply increases as $p$ grows larger than 0.45 . The average increases again as $\alpha$ grows to $\alpha=7$ in Figure 7(e), with the average predator population density lying between $\bar{P}=0.83$ to $\bar{P}=1.08$, with $\bar{P}>0.89$ for the vast majority of the tested space. A small window between $p=0.2-p$ $=0.22$ sees $\bar{P}$ drop discontinuously to 0.83 . The mutation rate here is very heavily impacting the average population density of the predator. In Figure 7(f) with $\alpha=\infty$, the average predator population density lies between $\bar{P}=1.1$ and $\bar{P}=1.39$. The behaviour here is very erratic, especially when $p>0.3$, where the output looks to be jumping between two different attractors as suggested when discussing Figure 3(f).

Looking at the prey with the lowest $r$ value, $r=0.3$, which corresponds to the graphs presented in Figure 4, as noted earlier a clear influence of the effect of mutation can be seen, especially with $\alpha \leq 2$. In Figure 7(a) where $\alpha=0$, a gradual increase of this species population between $N_{l}=0.56$ at $p=0.01$ and $N_{l}=0.73$ at $\underline{p}=0.5$ can be seen. Similar behaviour in this species is also seen in Figure 7(b) and 7(c) and 7(d) but with the difference becoming less clear as $\alpha$ increases. As $p$ increases, the contribution from prey species with higher growth rates and higher population density increases, which for these low values of $\alpha$ allows for more contribution from neighbouring species with higher current population density, causing an overall increase in the population density of $N_{l}$. With the higher values of $\alpha$ values presented in Figure 7(e) and 7(f), the species behaviour does not show as much reliance on mutation, with more significant variation apparent at $\alpha=7$.

For prey with $0.3<r<2.1$ the mutation rate and $\alpha$ have less impact on the prey species average survival rate. For the species where $r>2.1$, where the species' underlying dynamics can display chaotic behaviour covering a wide range of values, non-smooth behaviour of the average population density can be seen as $p$ varies. 
Figure 8 shows bifurcation diagrams with $\alpha$ varying between 0 and 10 and with a fixed $p$ value. Figure $8\left(\right.$ a) and $8\left(\right.$ b) use a fixed $p$ value of 0.41 , showing the predator and $N_{l}$ prey respectively. $p=0.41$ has been chosen since it is a point which sees survival for the full range of $c$ values where co-survival is seen, observable in Figure 3(a), at which the predator is surviving between $c=1.5$ and $c=4.5$. It is clear that varying the value of $\alpha$ impacts the underlying dynamics of the ecosystem, with the various bifurcation diagrams showing a full range of chaotic dynamics, with clear periods of periodic behaviour within the chaos for all values of $c$. As noted when discussing Figure 3, increasing the value of $\alpha$ is decreasing the highest $c$ for which co-survival is seen. This is clearly apparent here, for $c=4.5$, the predator is surviving up to $\alpha=0.04$, for $c=4$ the predator is surviving up to $\alpha=0.36$, for $c=3.5$ the predator is surviving up to $\alpha=0.14$, for $c=3$ the predator is surviving up to $\alpha=3.16$ and for $c=2.5$ the predator is surviving up to $\alpha=5.68$. The range of the chaotic attractor for these examples is increasing as $\alpha$ increases. With $c=2$ and $c=1.5$, the predator is surviving for the full range of $\alpha$ between 0 and 10. The $\alpha$ value at which the $c=1$ first survives can be seen as $\alpha$ $=1.6$.

The impact of $\alpha$ on the prey species can be seen in Figure 9, which shows the average population survival of the species in the ecosystem with $p=0.41$ and $\alpha$ varying between 0 and 10. Figure 9(a) shows the species' average behaviour with $c=1.5$. The average predator population density here is increasing as $\alpha$ increases. It is having a greater impact on those species where $r \geq 2.7$, with their average population decreasing as $\alpha$ increases. These are the two species with the widest range to their populations, and therefore the species that can have the highest maximum value. Although their average value is being reduced, the predator is spending most of its time predating upon them, impacting them to a greater degree. The other species have less range to their populations, with some species' average population density slightly increasing as $\alpha$ increases and some slightly decreasing. Figure 9(b) shows the species average' behaviour with $c=2$. More interesting dynamics are displayed here, initially the system behaves in a similar manner, with those species with highest $r$ values being impacted and the predator population increasing most with $\alpha<6$. At $\alpha>6$ however the average predator population rapidly jumps from 0.9 to 1.1 . The impact upon the prey changes, with the $r=2.1$ prey becoming the species with the lowest average value, and with there being much less impact on the $r=3$ species. This behaviour coincides with a long period of stable periodic behaviour observable in Figure 8 in the $c=2$ plot between $\alpha=6.03$ to $\alpha=9.02$, 
where the system goes from behaving similarly to the $c=1.5$ plot to displaying markedly different dynamics. This suggests that the system is jumping onto a different attractor at this point.

\subsection{Bifurcation Diagrams Ricker Based Model}

Figure 10 presents bifurcation diagrams showing predator behaviour in the Ricker based predator-prey model for different values of $\alpha$. These results are directly comparable to the results shown in Figure 3 for the logistic based model. Figure 11 shows the corresponding bifurcation diagrams for the $N_{l}$ prey population for different value of $\alpha$. These results are directly comparable to Figure 4.

As noted when discussing Figure 2, the overall region of co-survival is narrower in the range of $c$ values in comparison to the logistic based model. Similar to the logistic based model and as noted when discussing Figure 3, as $\alpha$ increases the maximum $c$ for which co-survival occurs decreases. In Figure 10(a) where $\alpha=0$ the predator survives between $c=1$ and $c=5$ for at least some values of the mutation rate $p$, while survival occurs between $c=1$ and $c=$ 3.5 for $\alpha=1$ in Figure 10(b) and between $c=1$ and $c=2.5$ for $\alpha=2$ in Figure 10(c). In Figure 10(d), Figure 10(e) and Figure 10(f) where $\alpha=4,7$ and $\infty$ respectively, the predator survives between $c=1$ and $c=1.5$. This clearly illustrates what one would expect from a comparison of the species survival plots, that the column of predator survival is more restricted in the Ricker model than in the logistic model for $\alpha>0$. It is also of note that in all cases the co-survival area successfully begins at $c=1$, whereas in the logistic based example, survival was not seen at $c=1$ until $\alpha>1$.

As in the discussion of Figure 3 and Figure 4 for the logistic model, the results for the Ricker model in Figures 10 and 11 display a wide range of dynamics as the mutation rate changes, with an increasing value of $c$ increasing the predator's population density within the region of co-survival, which in turn decreases the prey population densities as the predator becomes more effective at predating upon them. One difference between the models is that for $\alpha>0$ the Ricker model tends to explore a greater range of the phase space. This is particularly evident for $\alpha=1$ with the Ricker populations visiting the full range of 0 to 3.2, whereas under the logistic model the population values are restricted to lie between 0.35 and 2. Another interesting feature of the Ricker results can be seen in Figure 10(d) which provides a nice example of an attractor consisting of two distinct, but overlapping regions of phase space for $\alpha=4$ and $c=1.5$. In some cases mentioned in Figure 3(a) and Figure 3(b) it was observed 
that increasing the mutation rate could have a stabilising effect on the ecosystem, where the system period halved out of chaos and behaved periodically for all remaining values of $p$. This behaviour is also observed in the Ricker based model in Figure 10, for example, at $c=2$ for $\alpha=0$ and $\alpha=1$ and at $c=1.5$ for values of $\alpha$ from 0 to 4 . This behaviour is also evident within the bifurcation diagrams for the first prey species, $N_{l}$, in Figure 11.

The results in Figure 11(a) for $\alpha=0$ are very similar to those obtained using the logistic model which were presented in Figure 4(a). In general, the behaviour remains fairly similar to that in the logistic case for higher values of $\alpha$ and, in particular, the trend of the population decreasing with predation rate $c$ for a given value of $\alpha$ is evident. For $\alpha=1,2$, for each value of $c$, the prey starts in an apparently chaotic region, collapses to a low-period orbit, and then bifurcates back to chaos as the mutation rate is increased from zero. When $\alpha=\infty$ the first species has very little fluctuation in its population and remains close to a value of one, the value it would have in the absence of predation, except for very high mutation rates $(p>$ 0.48). As well as being distinct from the cases where $\alpha$ is finite, the results for $\alpha=\infty$ are distinct from the logistic case. While the population remains close to one where it survives, there is no evidence of it becoming extinct and then being brought back into existence as the result of mutation from the neighbouring prey species $N_{2}$ as there was in the logistic case.

Figure 12 shows a plot of the average population density of the predator and each of the prey with the Ricker based predator-prey model and $c=1.5$, with the $p$ control parameter being varied and with the use of various values of $\alpha$. This figure is comparable to Figure 7 which shows a similar plot for the logistic based model, but note that a different $c$ value of 1.5 is used in Figure 7 since this corresponds to a region of co-survival (see Figure 2). Note that the average prey populations are in general smoother and less variable in $p$ in comparison to the corresponding results obtained for the logistic model. A further difference compared to the logistic case is that in the range of low $p$, corresponding to the wider region of predator survival in the survival plots (Figure 2), the average population of the predator is much larger than for large $p$ and in some cases it is larger than that of some or even all of the prey species for $\alpha>1$. However, when $p$ increases beyond this range, the predator population falls significantly and there is often a discontinuity in the prey populations as they align themselves with a (usually lower) average population value which then remains relatively unchanged as $p$ continues to increase from that point onward. The critical value of $p$ at which this change occurs increases slightly with increasing $\alpha$ (for $\alpha=0$ it is approximately $p=0.11$, 
but by $\alpha=4$ it is $p=0.17$ ). This critical value of $p$ is due to bifurcations causing a rapid onset of chaos in the structure of the system observed in the corresponding predator and first prey species bifurcation diagrams for $\mathrm{c}=1.5$.

Figure 13 shows how increasing the value of $\alpha$ affects the dynamics of the model with a high mutation rate of $p=0.49$. This value of $p$ was chosen since it permits survival at higher values of $c$ at $\alpha=0$ (see Figure 2). Once again, the results are similar to those presented for the logistic model in Figure 8 (at $p=0.41$ ), although survival occurs for higher $c$ values in the Ricker model. Figure 13(a), which shows the behaviour of the predator, reinforces our earlier observation that increasing the value of $\alpha$ decreases the maximum value of $c$ that allows predator survival. As $\alpha$ increases, the maximum predator population size that is visited (in the case of periodic or chaotic behaviour) for a fixed value of $c$ will increase until the population visits a negative value whilst in the chaotic region and the predator thus immediately goes extinct. The plot continues with the next highest value of $c$, which although also increasing with $\alpha$ will have a lower population at that point.

Figure 13(b) shows the corresponding bifurcation diagram for the first prey species, $N_{l}$. It should be noted that in the event of the single predator going extinct, the simulation is halted. Thus, when the bifurcation diagrams end abruptly for values of $c>1.5$, this is because the predator has died out. So this should not be taken as indicative of a collapse of the prey species. As one might expect from the previous discussion, as the predation rate $c$ decreases the simulations continue for greater values of $\alpha$ and allow for greater maximum populations of the prey. This corresponds to the behaviour found in the logistic model in Figure 8(b).

\subsection{Discussion of Results}

Throughout this paper a predator-prey model has been explored with a set of mutating prey that is being predated upon by a single predator. Results were presented and discussed using both the logistic and Ricker models as the discrete time maps to model the prey's dynamics.

Initially the area of survival within the $(c, \mathrm{p})$ space was presented for both the logistic and Ricker model with various values of $\alpha$ with the use of a single predator. Distinct regions of behaviour were noted in both models, with a focus being placed on the region of co-survival. In both the logistic and Ricker models little reliance on the mutation rate was seen for this region. In both models the simplest strategies $(\alpha=0$ and $\alpha=1)$ allowed co-survival for the 
greatest range of parameter choices. It was noted that increasing the value of $\alpha$ generally decreased the maximum value of $c$ for which co-survival is seen. Concerning the prey, a single predator will never successfully suppress the prey species survival rate below total prey species survival within the area of co-survival. In the space either total prey survival is seen or in some cases when the logistic model is used the predator manages to hunt the prey to complete extinction, causing full ecosystem collapse.

Bifurcation diagrams showing the underlying behaviour within the space were then shown with a varying mutation rate. Across all the values of $\alpha$, and with both models, a vast range of dynamic behaviour arises in such a complex system. The system itself includes prey species that in their uncoupled form would behave chaotically, but with coupling and mutation, instances of periodic behaviour in the predator and prey species were identified. It was shown that varying either the mutation rate or the predator's value of $\alpha$ can greatly change the dynamics of the ecosystem. In both the logistic and Ricker models, instances were identified where the mutation rate could have a stabilising effect on the ecosystem, although in markedly different ways between the two models. Within the area of co-survival, increasing the value of $c$ increased the predator's population size, and in turn caused a decrease in the population sizes of the prey.

\section{Conclusions}

This paper has reviewed a predator-prey model where a single predator hunts a set of mutating prey. At the heart of the multiple species predator-prey model is an ecosystem with heterogeneity amongst the prey, with different prey species that each have different population growth rates. The focus here was placed on both the underlying dynamics of the predators and how the assignment of the various ecosystem parameters affected the species survival rate of the predators and the prey- with two separate unimodal maps being used to govern the dynamics of the prey.

Various observations where made using this model. It was noted that the area of co-survival had little dependence on the mutation rate, although when investigating the underlying behaviour of the species there was a clear reliance, with the species able to display a wide range of dynamics as the mutation rate varied. Increasing the value of $\alpha$ was noted to generally decrease the maximum predation rate for which co-survival was possible. It was also observed that within the area of co-survival total prey species survival was guaranteed, 
the single predator was never able to suppress the prey populations due to repopulation from mutation. It was noted that within the co-survival area, increasing the predation rate of the predator increased the predator's population size while decreasing the population size of the prey species. A further study will be carried out expanding on this research, with a model that allows for a set of multiple predators that mutate on the set of mutating prey, where each of the predators have a separate value of $\alpha$ to allow heterogeneity amongst both the predators and the prey species.

\section{References}

[1] Murray JD. Mathematical biology I: An introduction, vol. 17 of interdisciplinary applied mathematics. (Springer, 2002)

[2] Elsadany, A. E. A., EL-Metwally, H. A., Elabbasy, E. M., \& Agisa, H. N.. Chaos and bifurcation of a nonlinear discrete prey-predator system. Computational Ecology and Software 2(3):169-180 (2012)

[3] Neubert, Michael G., and Mark Kot. The subcritical collapse of predator populations in discrete-time predator-prey models. Mathematical Biosciences 110(1): 45-66 (1992)

[4] Liu, X., Xiao, D.: Complex dynamic behaviours of a discrete-time predator-prey system, Chaos, Solitons \& Fractals 32: 80-94 (2007)

[5] He, Z., Lai, X.: Bifurcation and chaotic behaviour of a discrete-time predator-prey system, Nonlinear Analysis: Real World Application, 12: 403-417 (2011)

[6] He, Zhimin, and Xin Lai. Bifurcation and chaotic behaviour of a discrete-time predator-prey system. Nonlinear Analysis: Real World Applications 12(1): 403-417 (2011)

[7] Mullan, Rory, D. H. Glass, and Mark McCartney. Classification and Collapse In Predator-Prey Models. Artificial Intelligence and Cognitive Science 2011, 344-352 (2011)

[8] Taylor, R., Sherratt, J, and White,A. Seasonal forcing and multi-year cycles in interacting populations: lessons from a predator-prey model. Journal of Mathematical Biology 67(6): 1741-1764 (2012)

[9] Neubert M.G., Kot M., Lewis M.A.: Dispersal and Pattern Formation in a Discrete-Time Predator-Prey Model, Theoretical Population Biology 48: 7-43 (1995)

[10] Costa, A., Boone, C. K., Kendrick, A. P., Murphy, R. J., Sharpee, W. C., Raffa, K. F., \& Reeve, J. D. Dispersal and edge behaviour of bark beetles and predators inhabiting red pine plantations. Agricultural and Forest Entomology 15(1): 1-11 (2013)

[11] Rodrigues, Luiz Alberto Díaz, Diomar Cristina Mistro, and Sergei Petrovskii. Pattern formation in a spaceand time-discrete predator-prey system with a strong Allee effect. Theoretical Ecology 5(3): 341-362 (2012)

[12] Liao X, Ouyang Z, Zhou S. Permanence of species in nonautonomous discrete Lotka-Volterra competitive system with delays and feedback controls. J Comput Appl Math. 211(1): 1-10 (2008) 
[13] Khoshsiar Ghaziani R, Govaerts W, Sonck C. Resonance and bifurcation in a discrete-time predator-prey system with holling functional response. Nonlinear Analysis: Real World Applications 13(3): 1451-1465 (2012)

[14] Chen Q, Teng Z, Hu Z. Bifurcation and control for a discrete-time prey-predator model with holling-IV functional response. International Journal of Applied Mathematics and Computer Science 23(2): 247-261 (2013)

[15] Fan Y, Li W. Permanence for a delayed discrete ratio-dependent predator-prey system with holling type functional response. Journal of Mathematical Analysis and Applications 299(2): 357-374 (2004)

[16] Holling, C.S. Some Characteristics of Simple Types of Predation and Parasitism, The Canadian Entomologist 91: 385-398 (1959)

[17] Abrams, P.A., Ginzburg L.R. The nature of predation: prey dependent, ratio dependent or neither? Trends in Ecology and Evolution 15(8): 337 - 341 (2000)

[18] Allhoff, K.T., Drossel B. When do evolutionary food web models generate complex networks? Journal of Theoretical Biology 334: 122-129 (2013)

[19] McCann, K.S. The Diversity-Stability Debate. Nature 405: 228-233 (2000)

[20] May, R.M. Stability and complexity in model ecosystems (Princeton Univ. Press, 1973)

[21] Grimm, V., Wissel C. Babel, or the ecological stability discussions: an inventory and analysis of terminology and a guide for avoiding confusion. Oecologia 109(3): 323-334 (1997)

[22] Dunne, J.A., Williams, R.J., Martinez, N.D. Network structure and biodiversity loss in food webs: robustness increases with connectance. Ecology Letters 5: 558-567 (2002)

[23] McCann, K., Hastings, A. and Huxel, G.R. Weak trophic interactions and the balance of nature. Nature 395(6704): 794-798 (1998)

[24] Mougi A, Iwasa Y. Unique coevolutionary dynamics in a predator-prey system. J Theor Biol. 277(1): 83$89(2011)$

[25] Mougi A. Predator-prey coevolution driven by size selective predation can cause anti-synchronized and cryptic population dynamics. Theor Popul Biol. 81(2): 113-118. (2012)

[26] Durrett R, Mayberry J. Evolution in predator-prey systems. Stochastic Processes and their Applications. 120(7): 1364-1392. (2010)

[27] Loeuille, N., Loreau, M. Evolutionary Emergence of Size-Structured Food Webs. PNAS 102 (16): 5761$5766(2005)$

[28] Caldarelli, G., Higgs, P.G., McKane, A.J. Modelling Coevolution in Multispecies Communities. J. Theor. Biol. 193: 345-358 (1998)

[29] Drossel, B., Higgs, P.G., McKane, A.J. The Influence of Predator-Prey Population Dynamics on the Longterm Evolution of Food Web Structure. J. Theor. Biol. 208: 91-107 (2001) 
[30] Yoshida, K. Evolutionary Dynamics of Species Diversity in an Interaction Web System. Ecological Modelling 163: 131-143 (2003)

[31] Rossberg, A.G., Ishii, R. Amemiya, T. and Itoh, K. The Top-Down Mechanism for Body-Mass-Abundance Scaling. Ecology 89(2): 567-580 (2008)

[32] Allhoff, K.T., Ritterskamp, D., Rall, B.C., Drossel, B. and Guill, C. Evolutionary Food Web Model based on Body Masses gives Realistic Networks with Permanent Species Turnover. Sci. Rep. 5: 10955 (2015)

[33] Drossel, B., McKane, A.J. Modelling Food Webs. Handbook of Graphs and Networks, 218-247 (Wiley$\mathrm{VCH}, 2003)$

[34] Petrovskii SV, Malchow H. A minimal model of pattern formation in a prey-predator system. Math Comput Model 29(8):49-63 (1999)

[35] Zhang L. Spatiotemporal patterns in a ratio-dependent food chain model with reaction-diffusion. Abstract and Applied Analysis 2014: Article ID 130851. Doi: 10.1155/2014/130851 (2014)

[36] Santinath P, Anindita T. Spatiotemporal dynamics of two dimensional logistic maps over a complex network. International Journal of Applied Mathematical Research. 1(4):604-610. (2012)

[37] Lopez-Ruiz R, Fournier-Prunaret D. Three logistic models for the two-species interactions: Symbiosis, predator-prey and competition. arXiv preprint nlin/0406020 (2004).

[38] Rodrigues LAD, Mistro DC, Petrovskii S. Pattern formation, long-term transients, and the Turing-Hopf bifurcation in a space-and time-discrete predator-prey system. Bull Math Biol. 73(8): 1812-1840 (2011)

[39] Kaneko, K. "Simulating physics with coupled map lattices" in Formation, Dynamics and Statistics of Patterns, vol. 1, eds. Kawasaku, K., Suzuki, M., Onuki, A. (World Scientific, Singapore 1990)

[40] Buminovich, L.A., Sinai, Y.G. Spacetime chaos in coupled map lattices. Nonlinearity 1: 491-516 (1988)

[41] Hendry, R.J., McGlade, J.M., Weiner, J. A coupled map lattice model of the growth of plant monoculture. Ecological Modelling 84: 81-90 (1996)

[42] Hassell, M.P., Comins, H.N., May, R.M. Spatial structure and chaos in insect population dynamics. Nature 353: 255-258 (1991)

[43] Solé, R.V., Bascompte, J., Vails, J. Nonequilibrium dynamics in lattice ecosystems: chaotic stability and dissipative structures. Chaos 2: 387-395 (1992)

[44] Mullan, Rory, David H. Glass, and Mark McCartney. "Species diversity and predation strategies in a multiple species predator-prey model." Commun. Nonlinear Sci. Numer. Simul. 25(1-3): 118-135 (2015) 


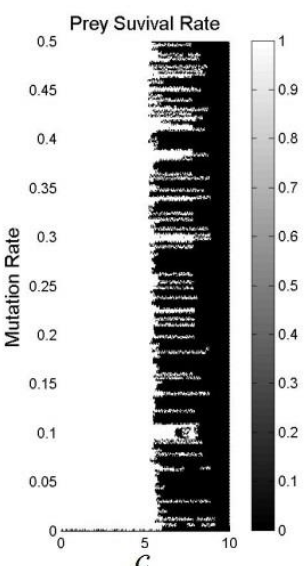

(a)

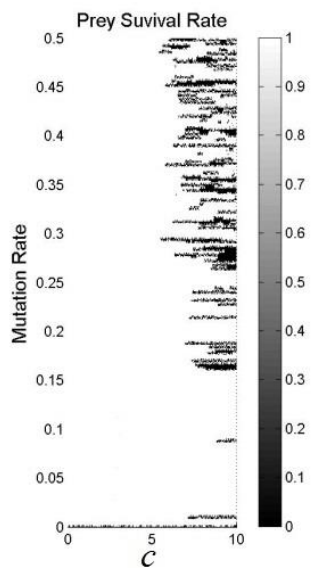

(c)

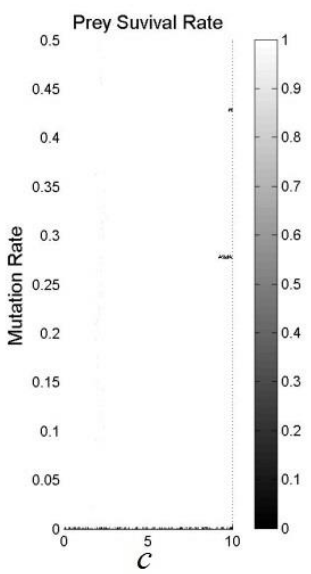

(e)
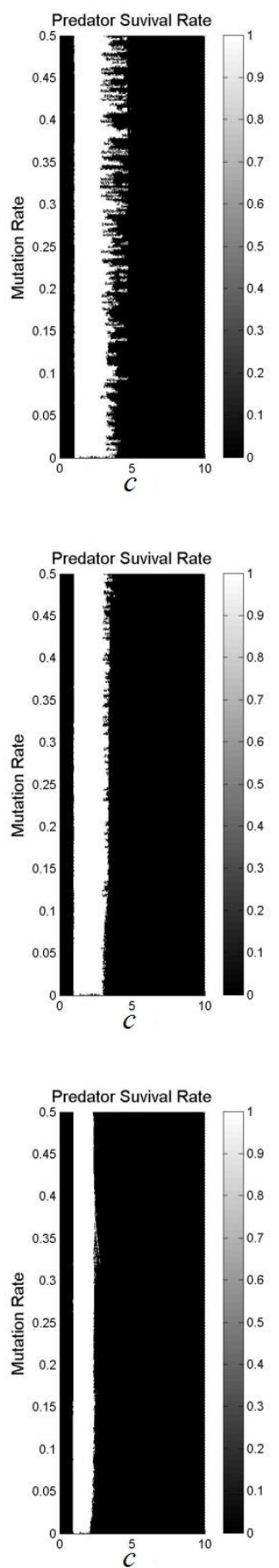

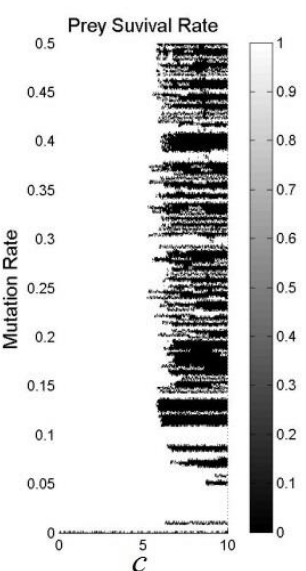

(b)
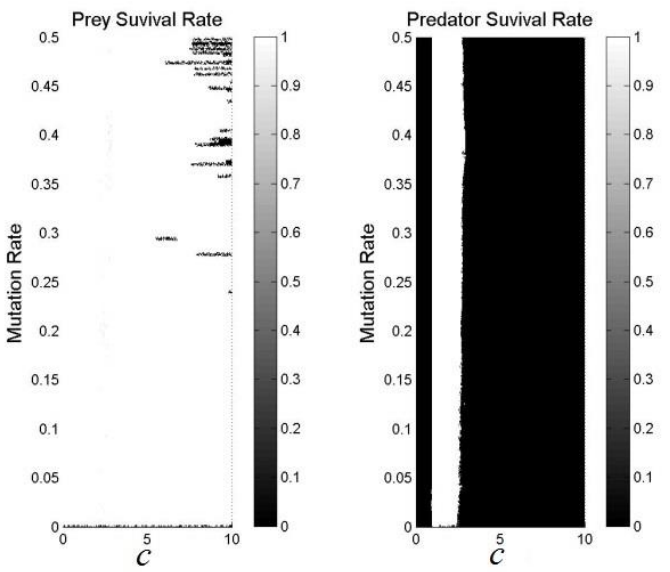

(d)
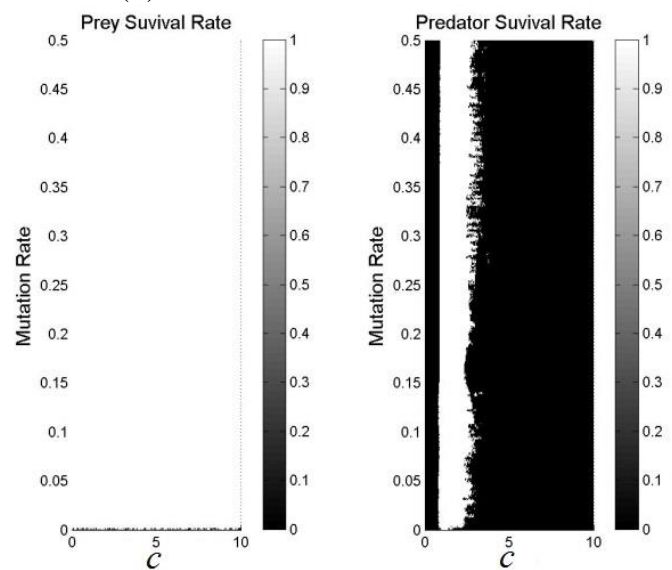

(f)

Figure 1. Survival rates showing the proportion of surviving species in a single predator, 10 prey, ecosystem with the use of the logistic map and scaled control parameter values. Results for different values of the focus strength, $\alpha$, are presented as follows: (a) $\alpha=0$, (b) $\alpha=1$, (c) $\alpha=2$, (d) $\alpha=4$, (e) $\alpha=7$ and (f) $\alpha=\infty$. 


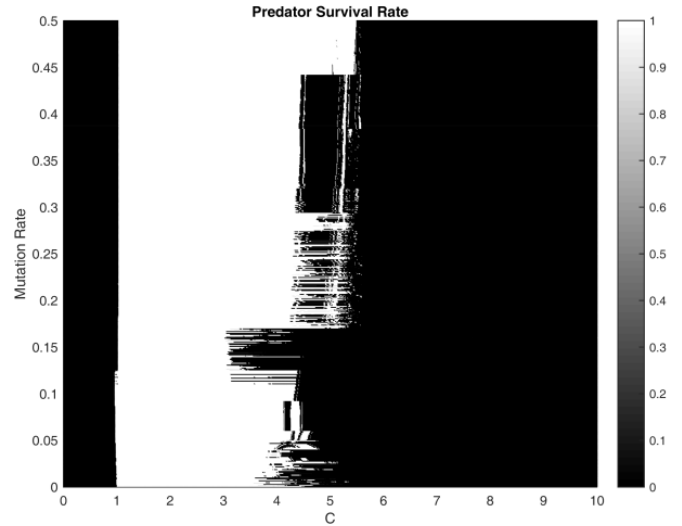

(a)

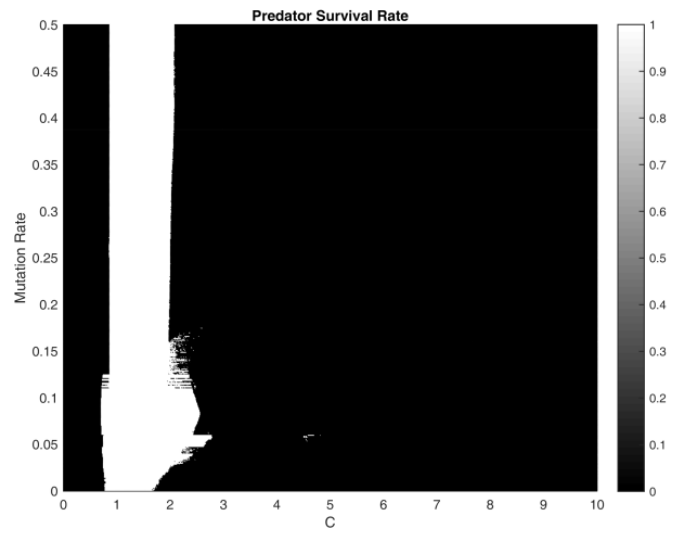

(c)

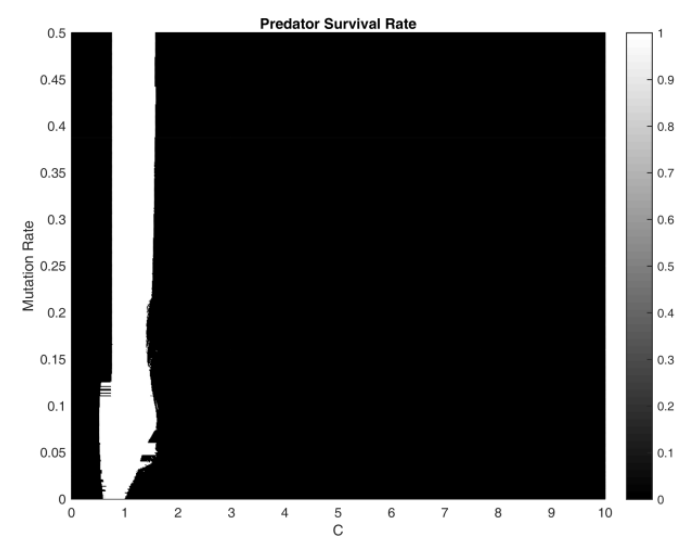

(e)

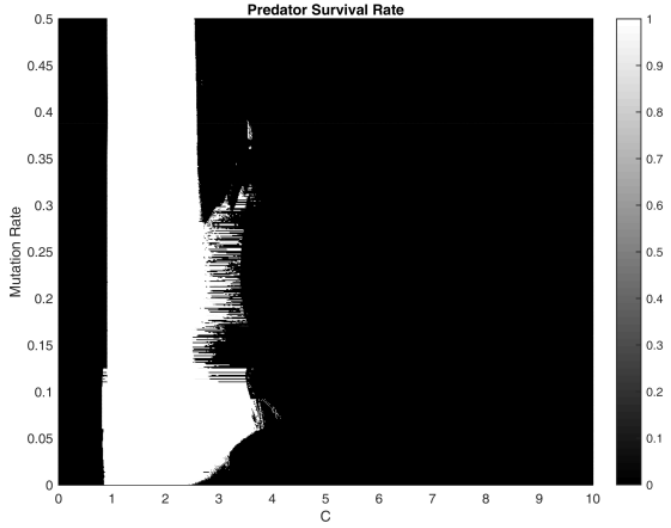

(b)

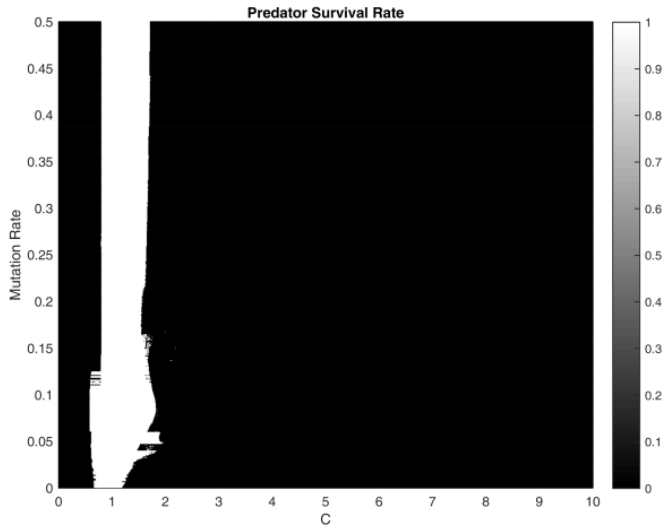

(d)

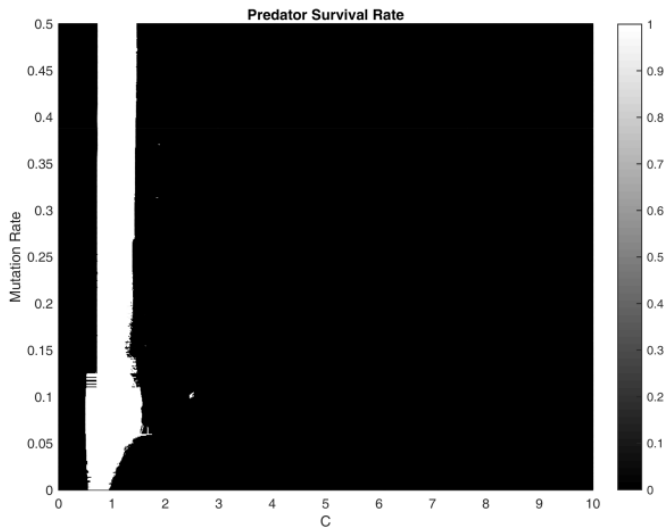

(f)

Figure 2. Predator survival rates for a single predator, 10 prey, ecosystem with the use of the Ricker map and scaled control parameter values. Results for different values of the focus strength, $\alpha$, are presented as follows: (a) $\alpha=0$, (b) $\alpha=1$, (c) $\alpha=2$, (d) $\alpha=4$, (e) $\alpha=7$ and (f) $\alpha=\infty$. 


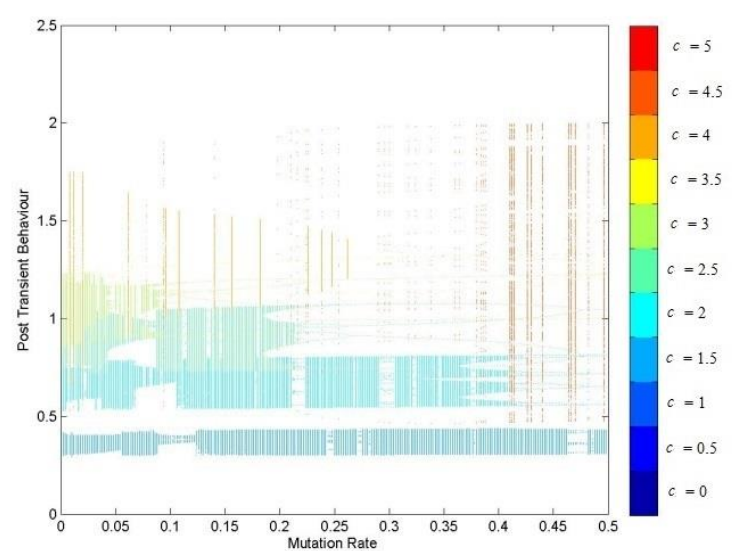

(a)

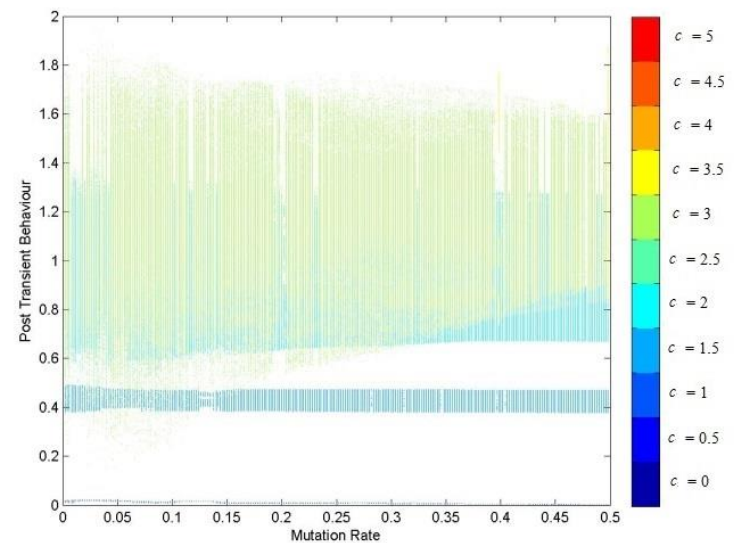

(c)

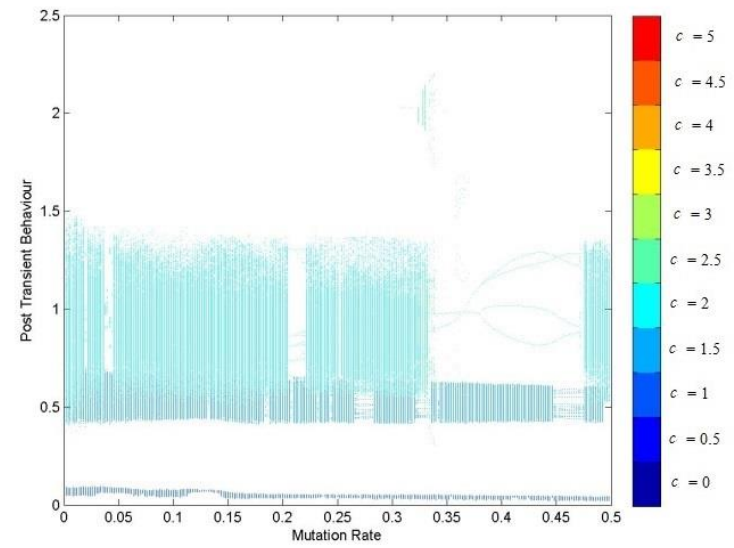

(e)

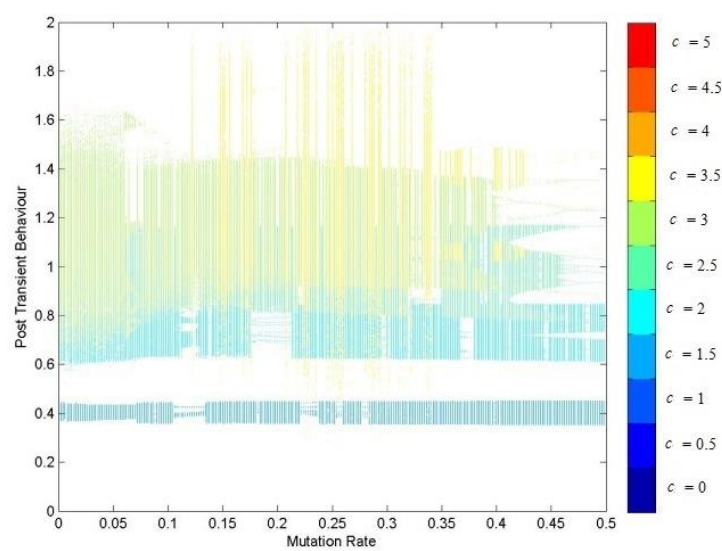

(b)

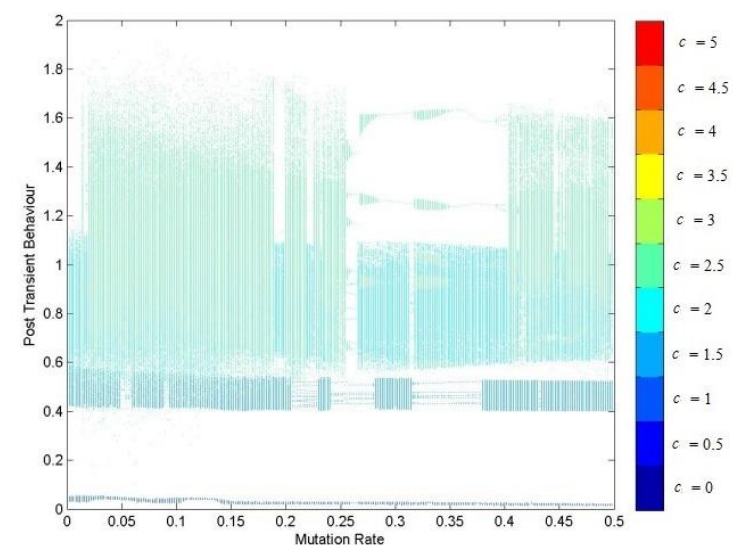

(d)

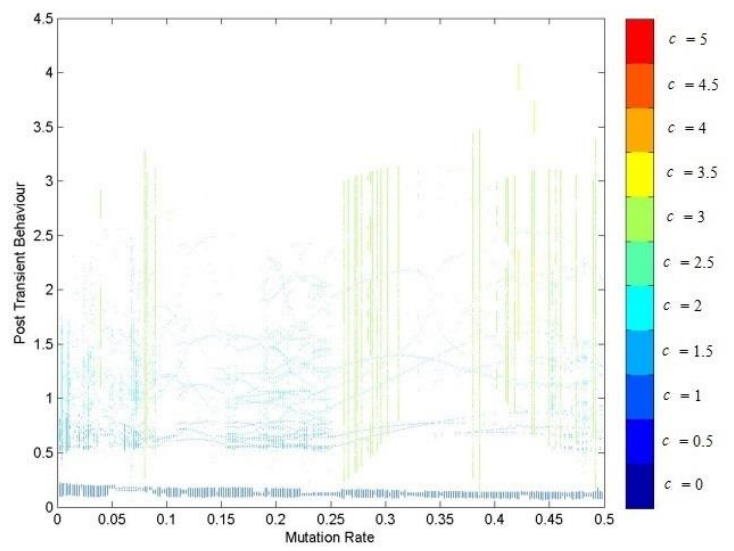

(f)

Figure 3. Bifurcation diagrams showing predator behaviour in the logistic map based predator-prey model. The plots show different $c$ values, with the mutation rate being varied. Results for different values of the focus strength, $\alpha$, are presented as follows: (a) $\alpha=0$, (b) $\alpha=1$, (c) $\alpha=2$, (d) $\alpha=4$, (e) $\alpha=7$ and (f) $\alpha=\infty$. 


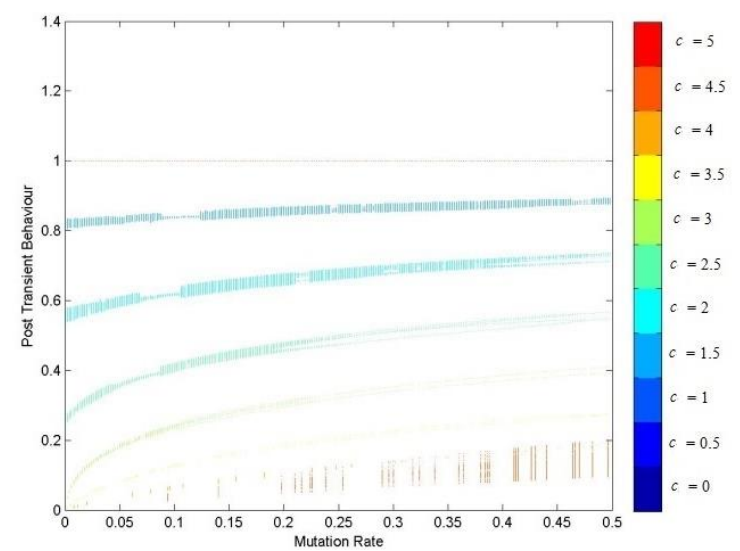

(a)

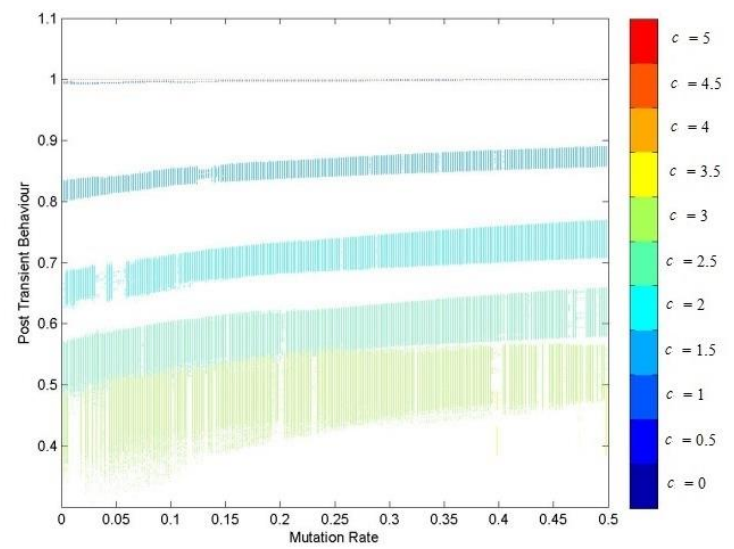

(c)

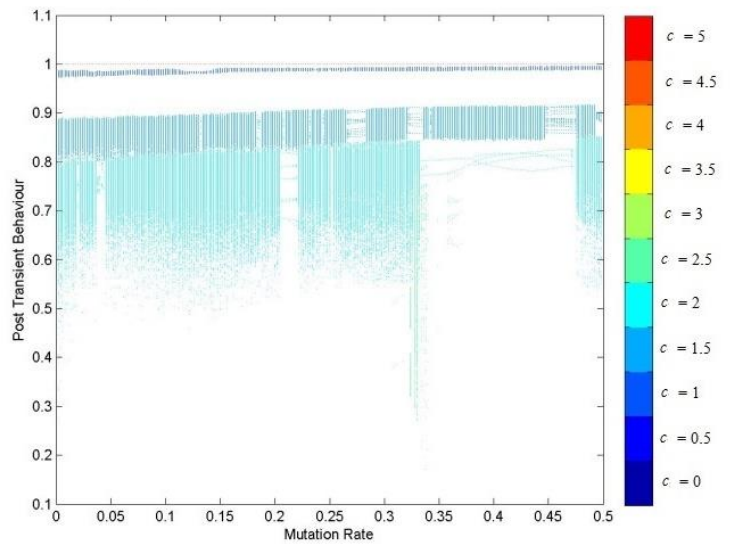

(e)

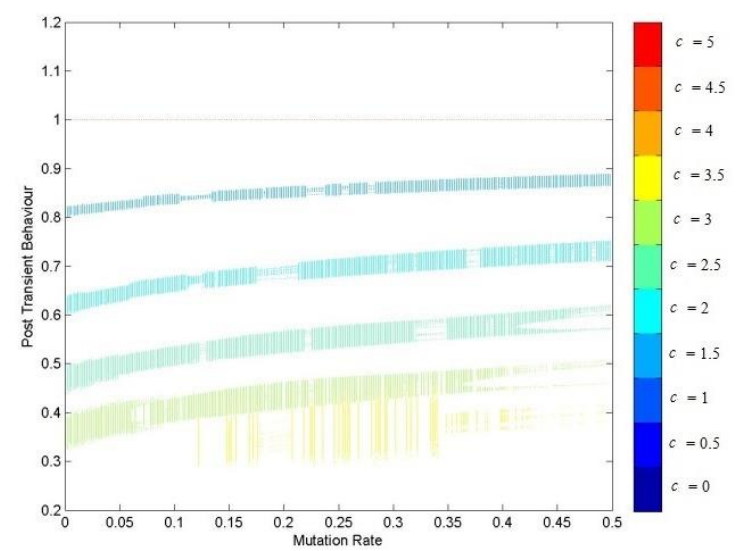

(b)

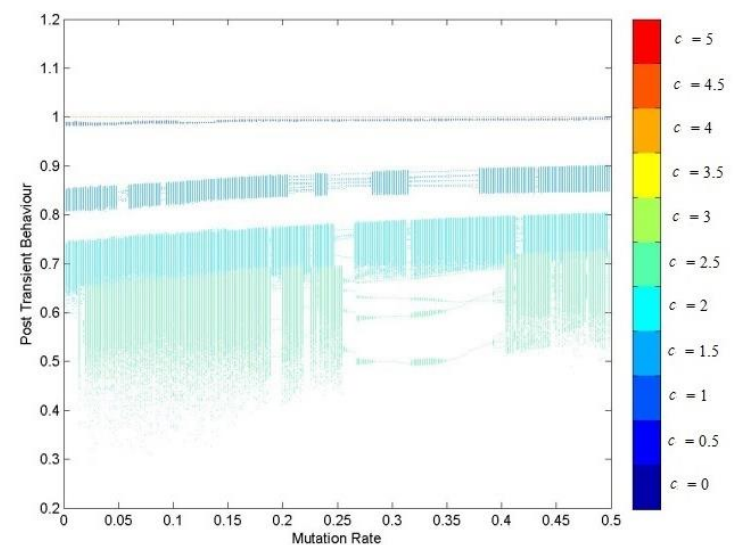

(d)

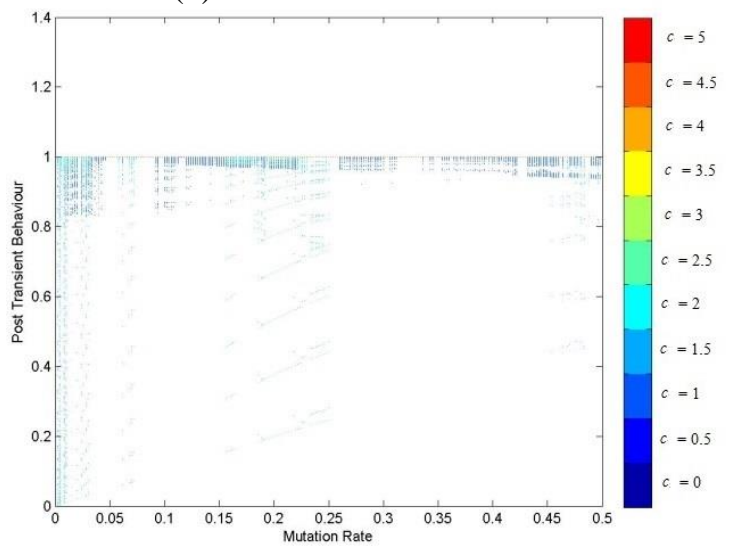

(f)

Figure 4. Bifurcation diagrams showing the behaviour of the $1^{\text {st }}$ prey species $\left(N_{l}\right.$, with $\left.r_{l}=0.3\right)$ in the logistic map based predator-prey model. The plots show different $c$ values, with the mutation rate being varied. Results for different values of the focus strength, $\alpha$, are presented as follows: (a) $\alpha=0$, (b) $\alpha=1$, (c) $\alpha=2$, (d) $\alpha=4$, (e) $\alpha=7$ and (f) $\alpha=\infty$. 


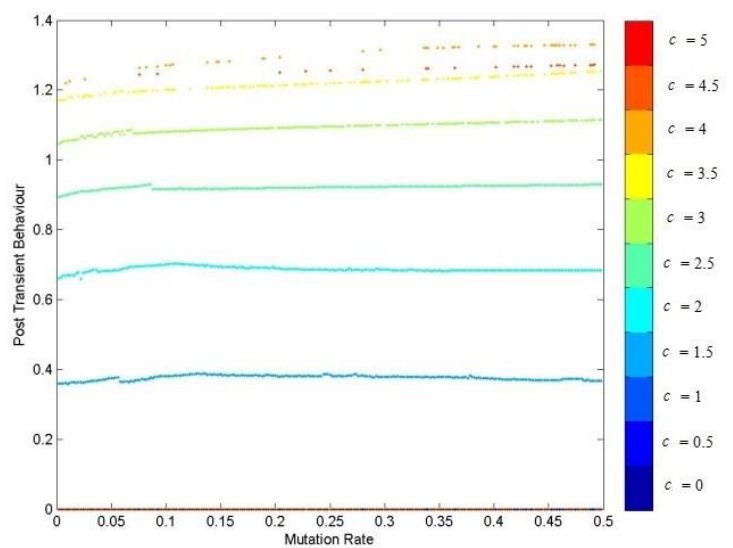

(a)

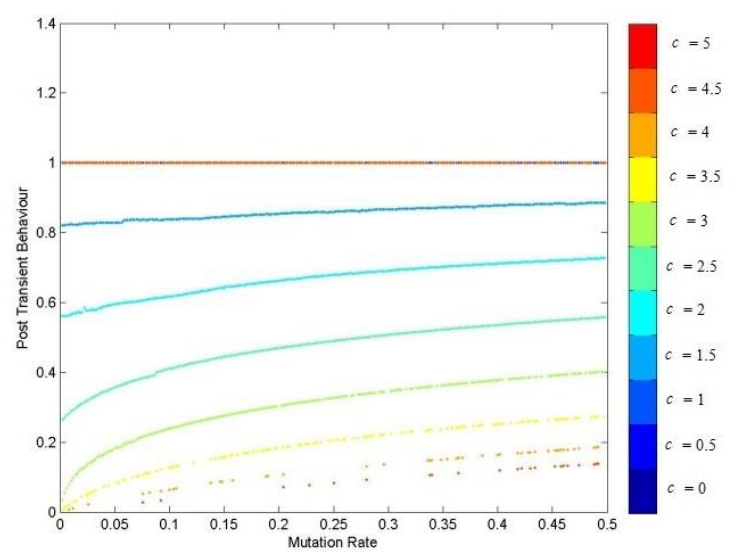

(b)

Figure 5. Averages for the predator and $1^{\text {st }}$ prey species $\left(N_{l}\right.$, with $\left.r_{l}=0.3\right)$ with an increasing value of $c$ and $\alpha$ $=0$ in the logistic map based predator-prey model, (a) shows the average population value of the predator species and (b) shows the average population value for the $N_{l}$ prey species. 


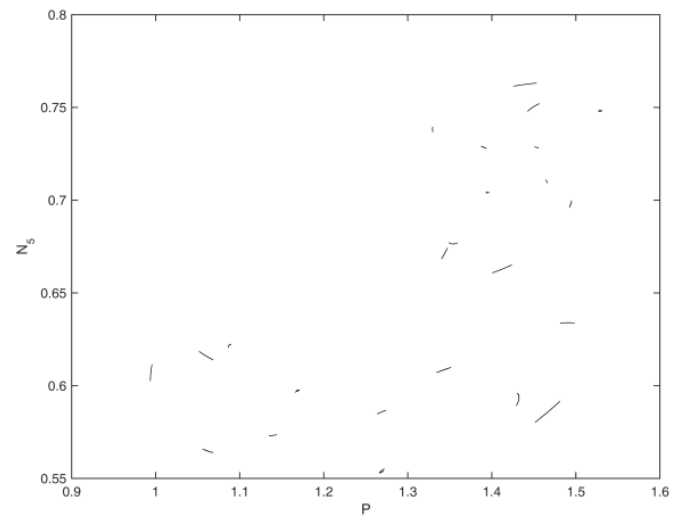

(a)

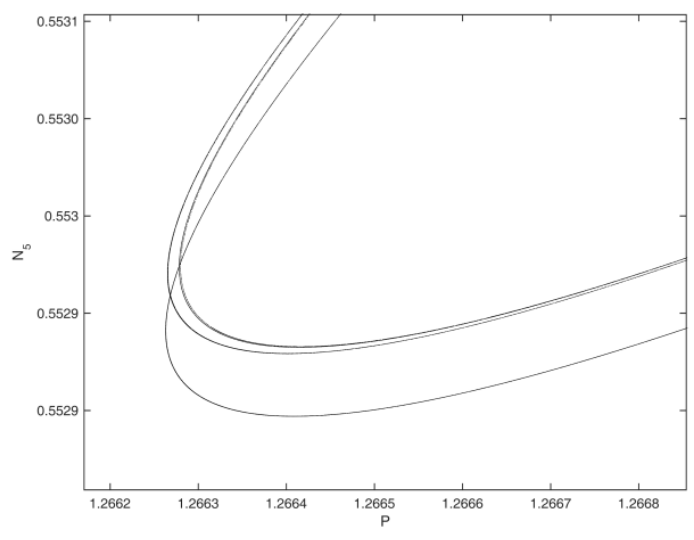

(b)

Figure 6. The dynamics in the logistic map based predator-prey ecosystem between the predator and the fifth prey species $\left(N_{5}\right.$, with $\left.r_{5}=1.5\right)$ with $\alpha=1, c=3.5$ and $p=0.355296$. (a) shows the full strange attractor, with $5 \times 10^{6}$ points displayed following $10^{7}$ transients, and (b) illustrates the structure with a zoom on the corner of the part of the attractor which has the lowest value of $N_{5}$ in (a). 


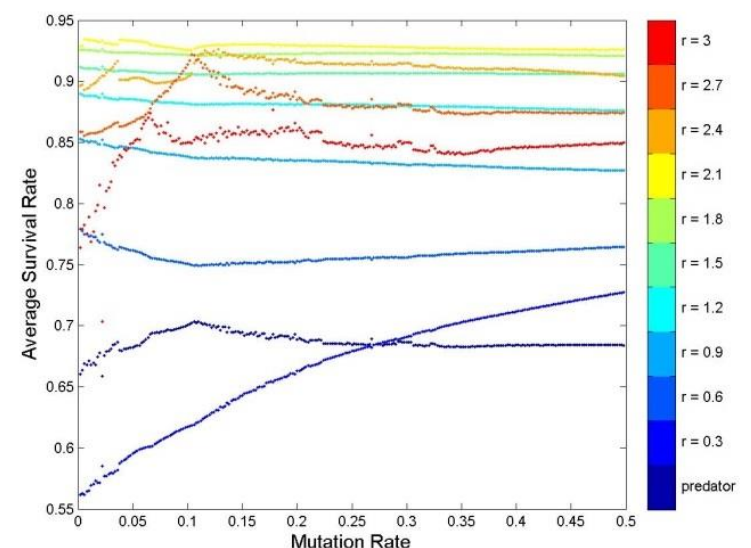

(a)

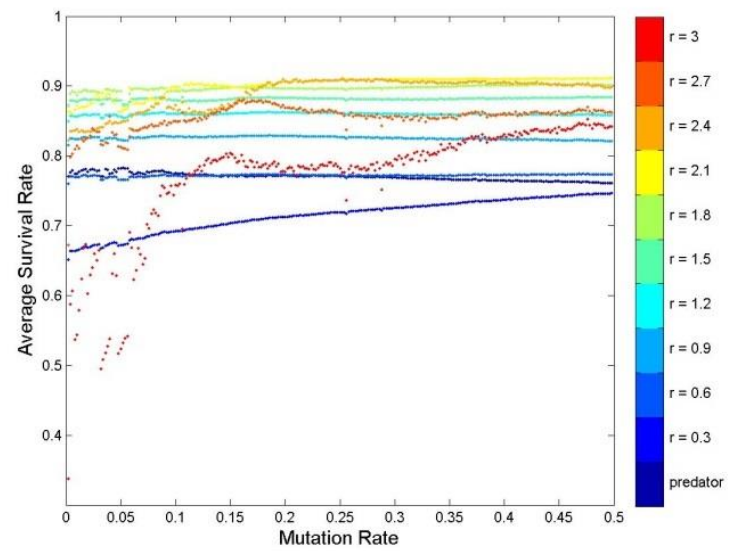

(c)

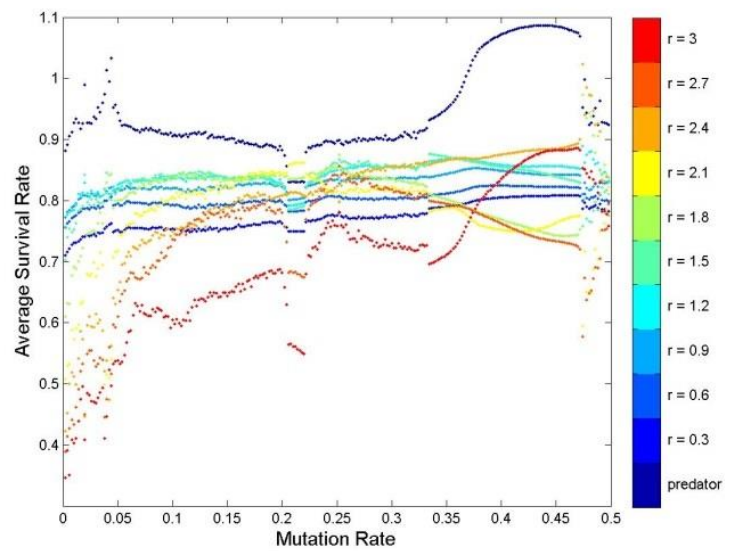

(e)

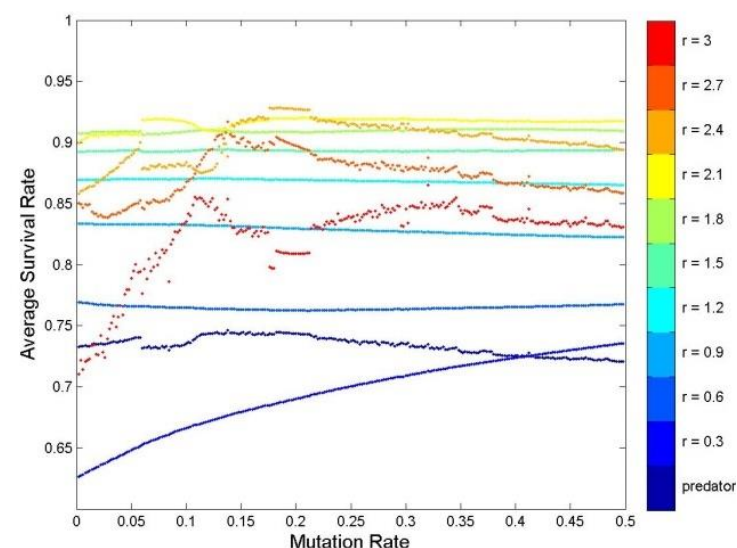

(b)

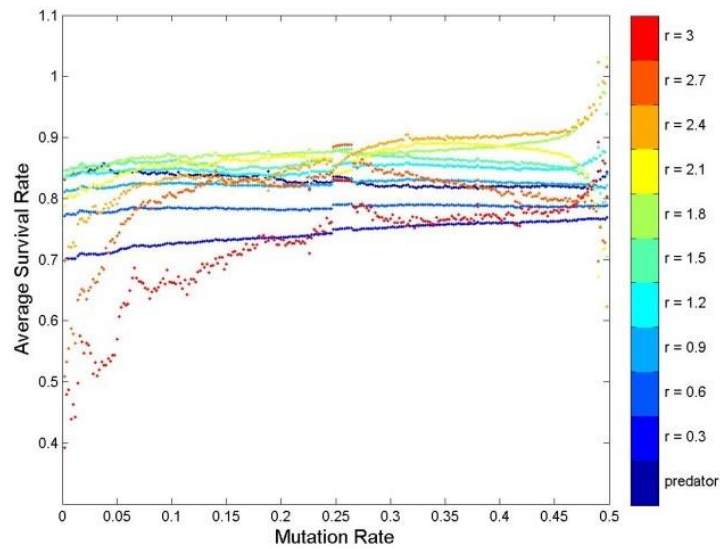

(d)

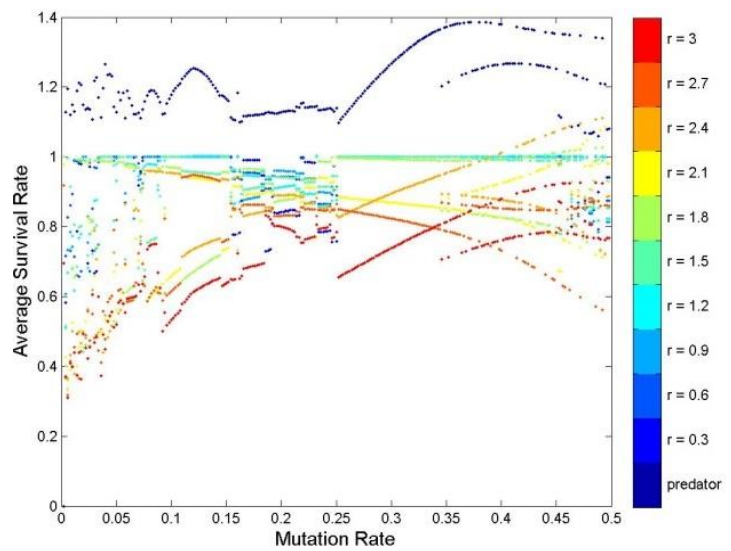

(f)

Figure 7. Average population density diagrams for the predator and each of the prey with the logistic map based predator-prey model with $c=2$. Results for different values of the focus strength, $\alpha$, are presented as follows: (a) $\alpha=0$, (b) $\alpha=1$, (c) $\alpha=2$, (d) $\alpha=4$, (e) $\alpha=7$ and (f) $\alpha=\infty$. 


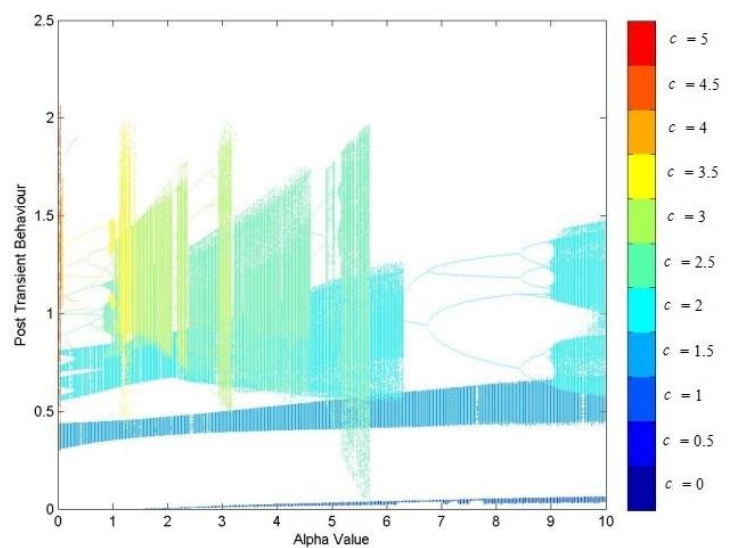

(a)

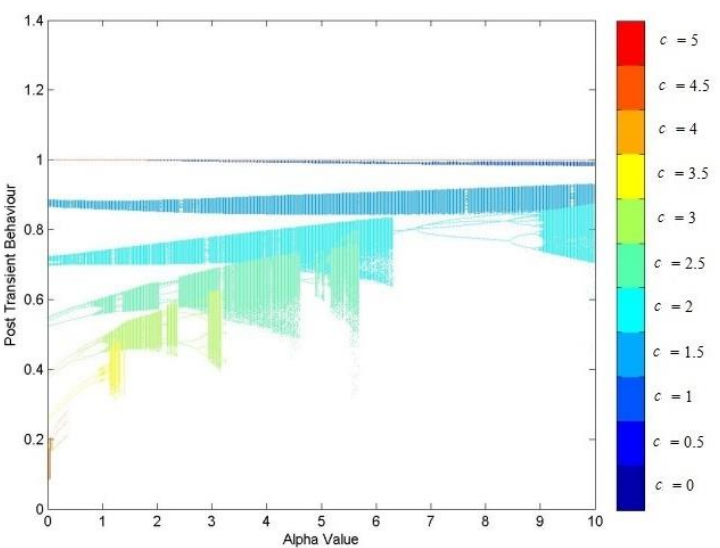

(b)

Figure 8. Bifurcation diagrams showing behaviour at fixed values of $c$ for varying $\alpha$ between 0 and 10 with a fixed mutation rate of $p=0.41$ for the logistic map based predator-prey model, (a) shows the predator's behaviour and (b) shows the $1^{\text {st }}$ prey species $\left(N_{l}\right.$, with $\left.r_{l}=0.3\right)$ behaviour. 


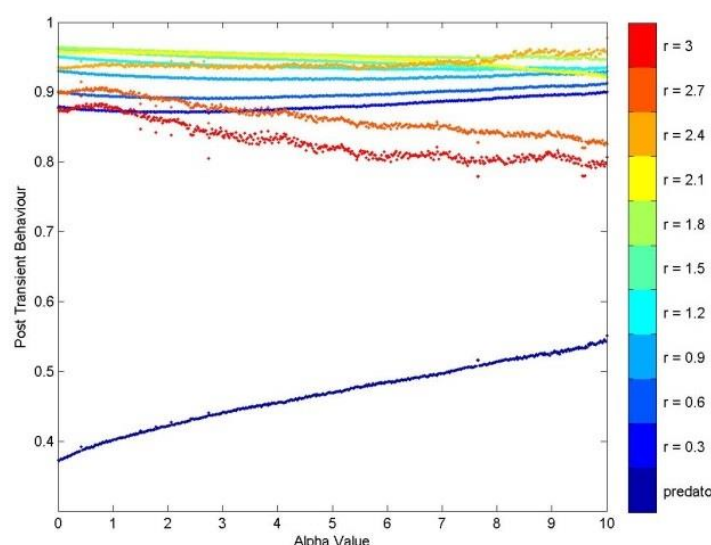

(a)

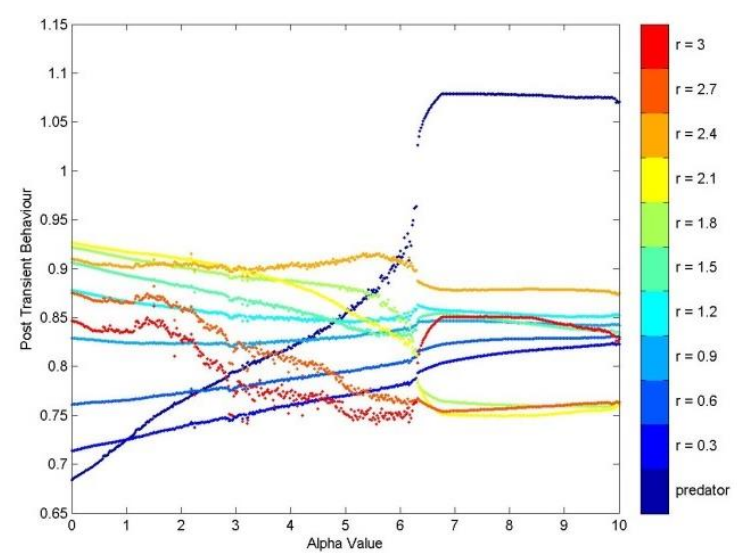

(b)

Figure 9. Average population survival of the species with $p=0.41$ and $\alpha$ varying between 0 and 10 for the logistic map based predator-prey model, (a) shows the species average behaviour with $c=1.5$ and (b) shows the average behaviour with $c=2$. 


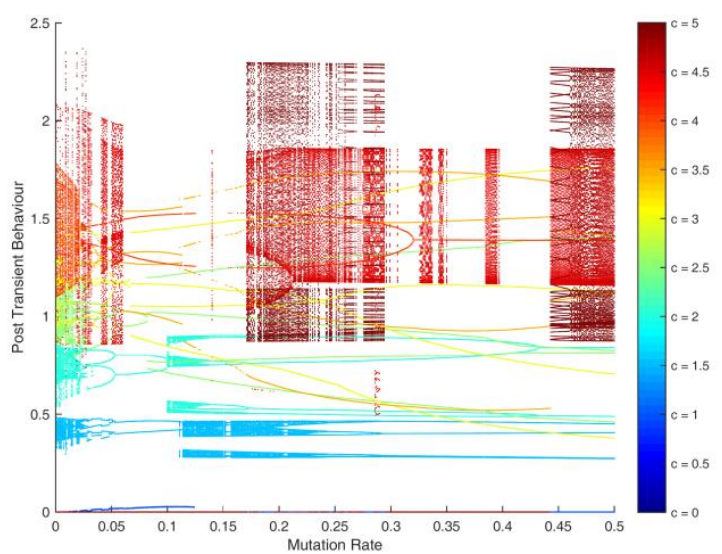

(a)

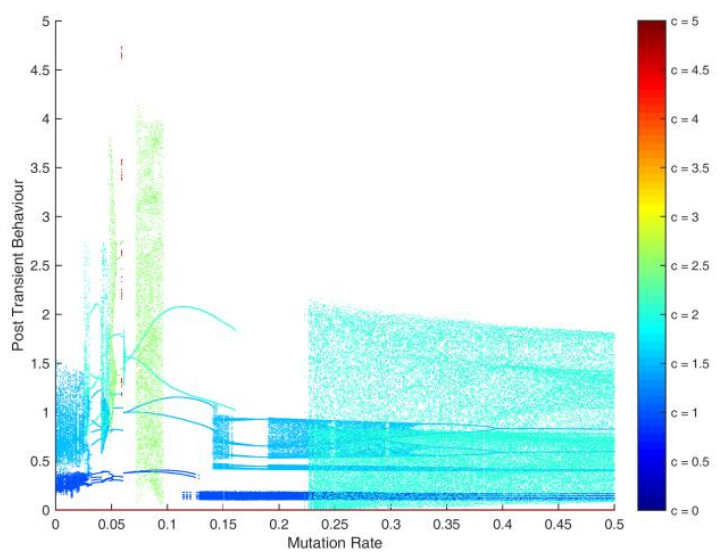

(c)

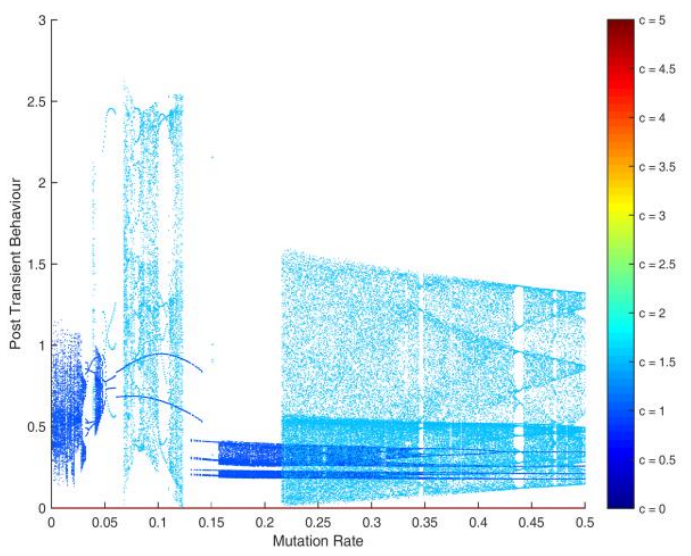

(e)

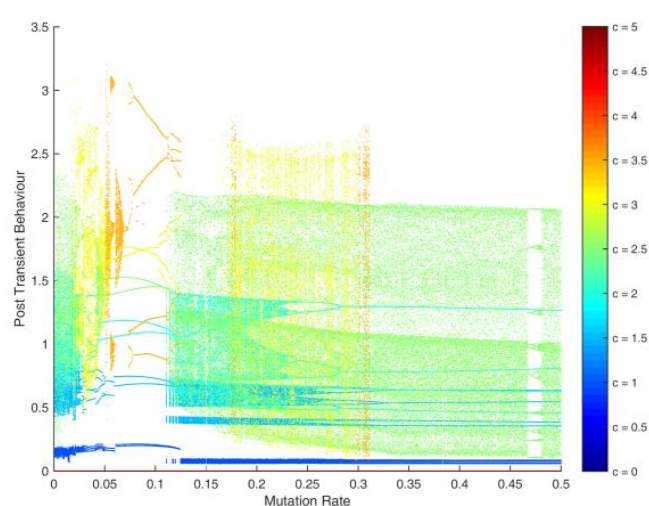

(b)

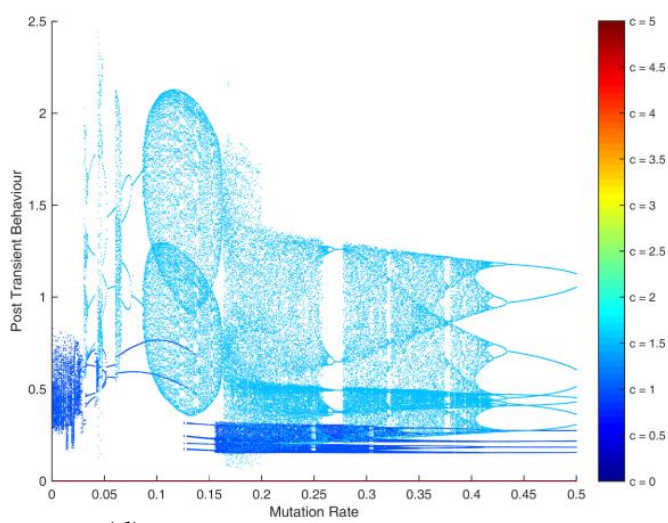

(d)

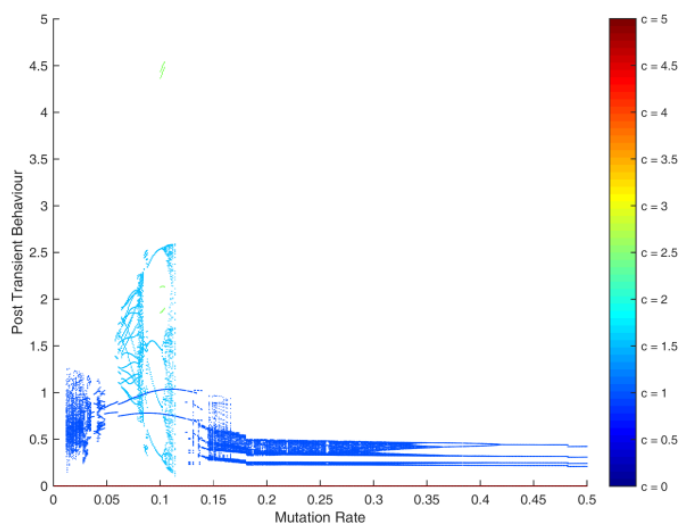

(f)

Figure 10. Bifurcation diagrams showing predator behaviour in the Ricker map based predator-prey model. The plots show different $\mathrm{c}$ values, with the mutation rate being varied. Results for different values of the focus strength, $\alpha$, are presented as follows: (a) $\alpha=0$, (b) $\alpha=1$, (c) $\alpha=2$, (d) $\alpha=4$, (e) $\alpha=7$ and (f) $\alpha=\infty$. 


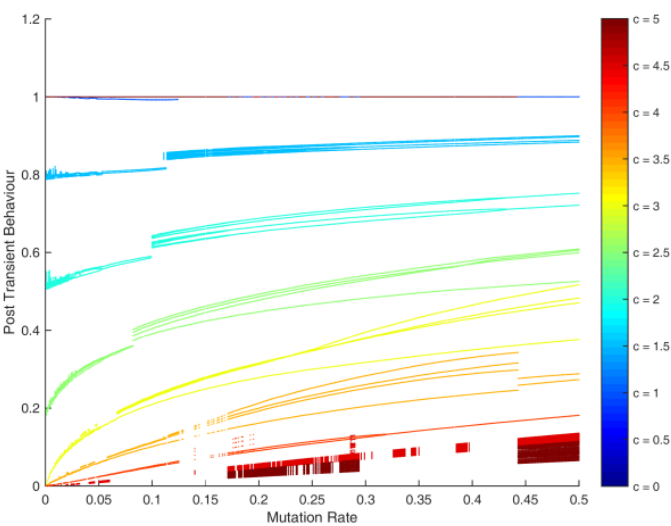

(a)

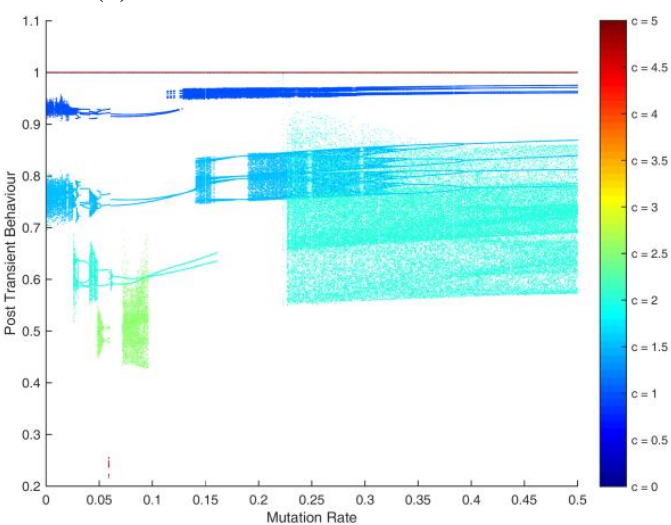

(c)

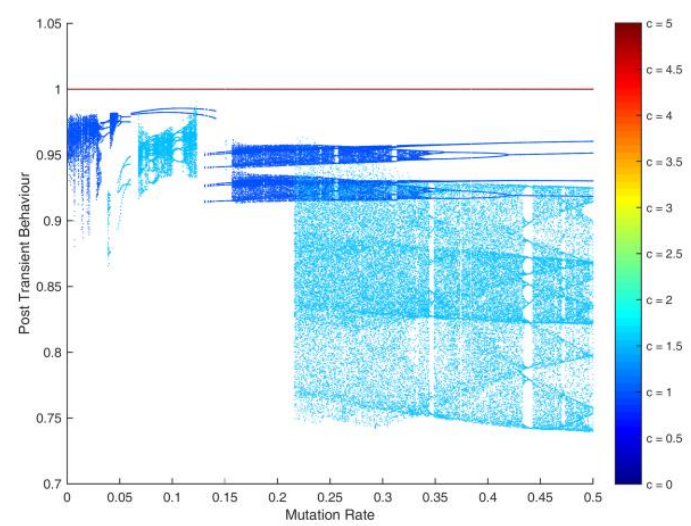

(e)

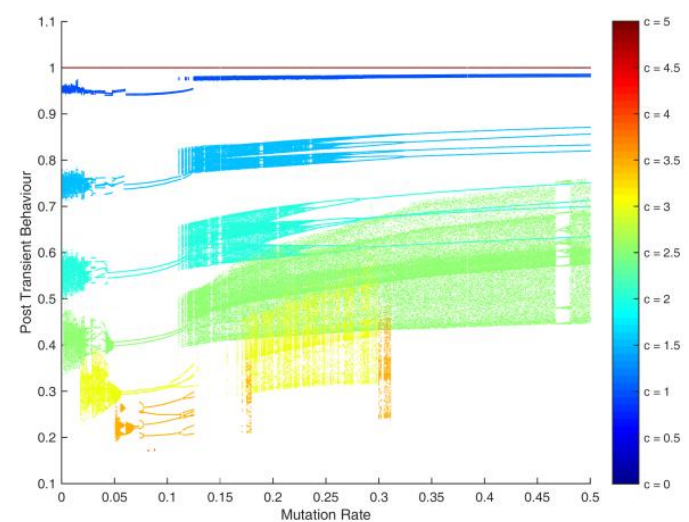

(b)

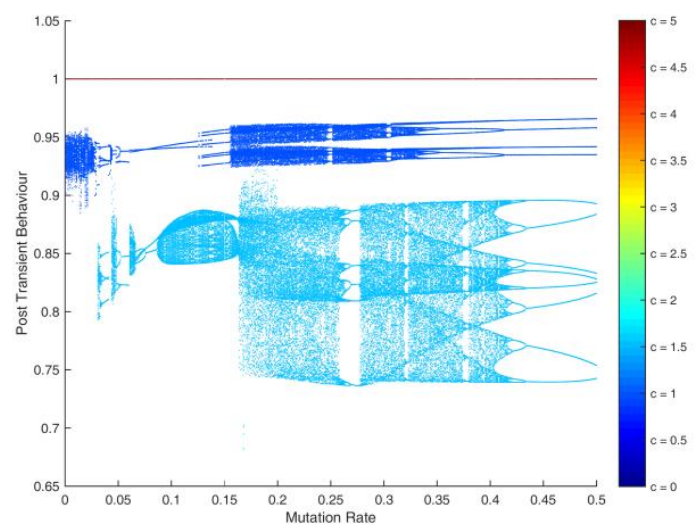

(d)

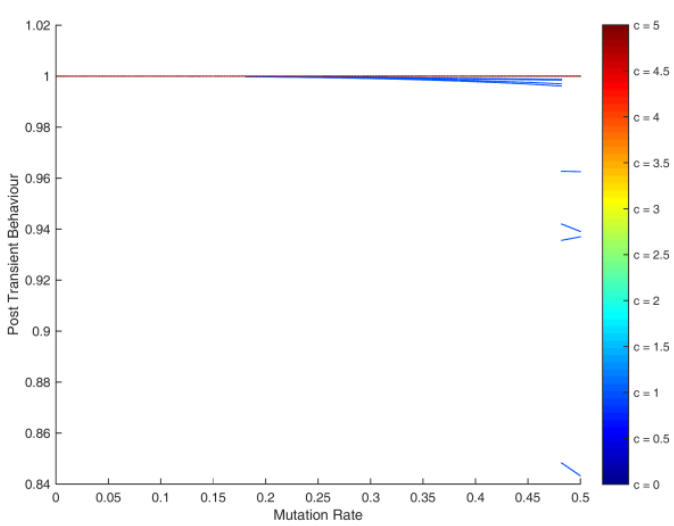

(f)

Figure 11. Bifurcation diagrams showing the $1^{\text {st }}$ prey species $\left(N_{l}\right.$, with $\left.r_{l}=0.3\right)$ behaviour in the Ricker map based predator-prey model. The plots show different c values, with the mutation rate being varied. Results for different values of the focus strength, $\alpha$, are presented as follows: (a) $\alpha=0$, (b) $\alpha=1$, (c) $\alpha=2$, (d) $\alpha=4$, (e) $\alpha=$ 7 and (f) $\alpha=\infty$. 


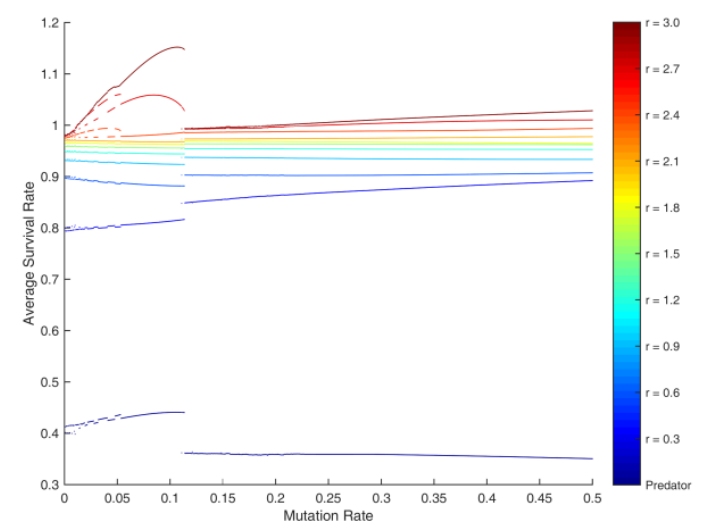

(a)

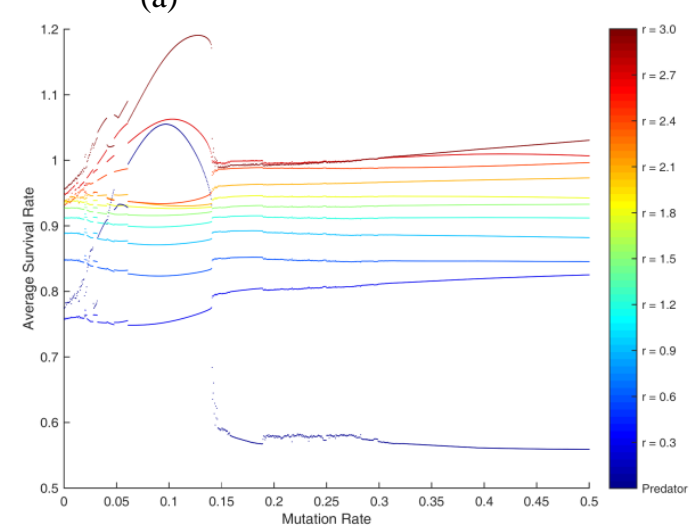

(c)

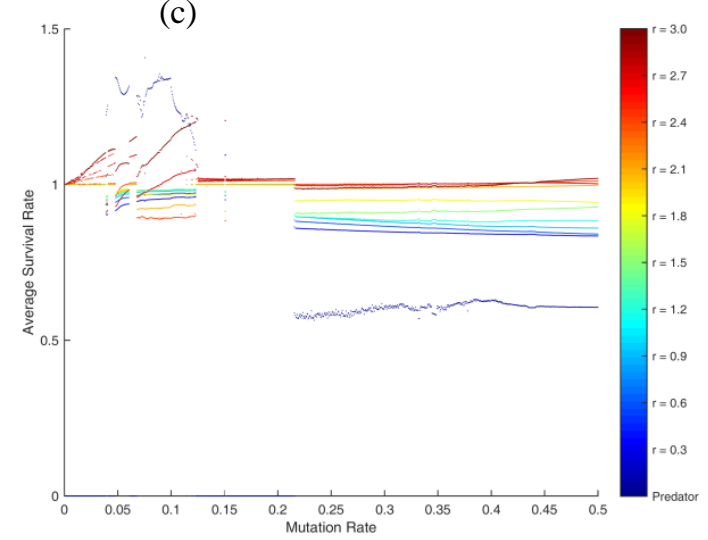

(e)

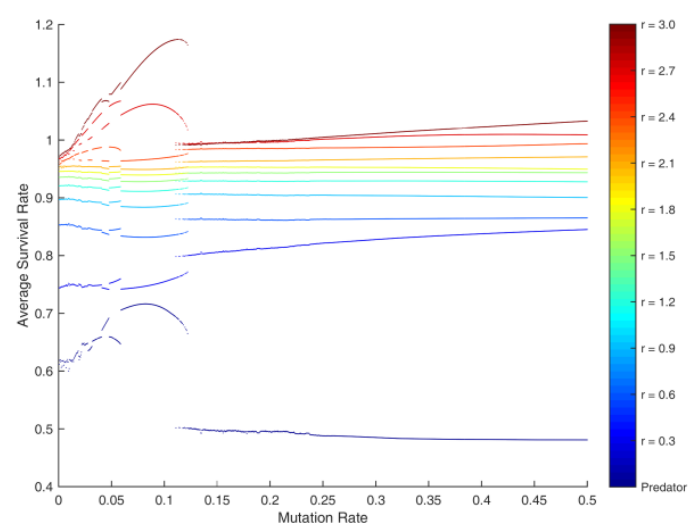

(b)

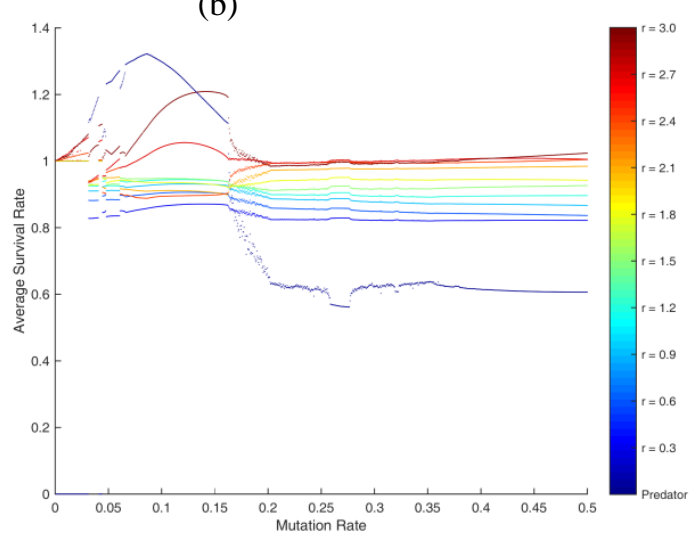

(d)

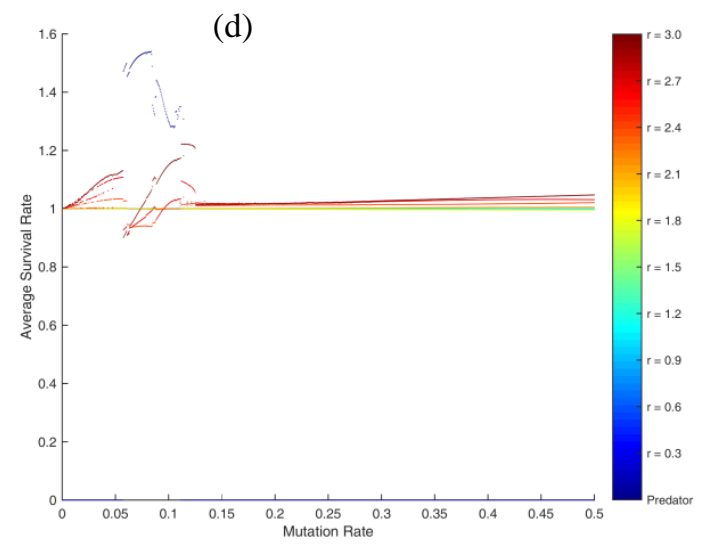

(f)

Figure 12. Average population density diagrams showing the average of the predator and each of the prey with the Ricker map based predator-prey model with $c=1.5$. The plots show different species, with the mutation rate being varied. Results for different values of the focus strength, $\alpha$, are presented as follows: (a) $\alpha=0$, (b) $\alpha=1$, (c) $\alpha=2$, (d) $\alpha=4$, (e) $\alpha=7$ and (f) $\alpha=\infty$. 


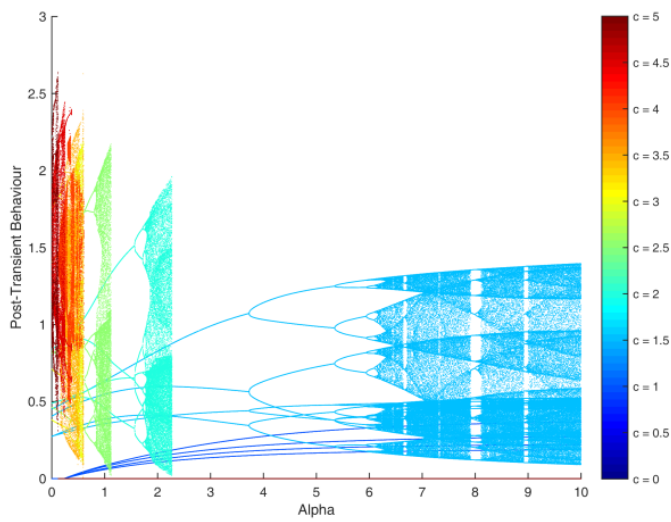

(a)

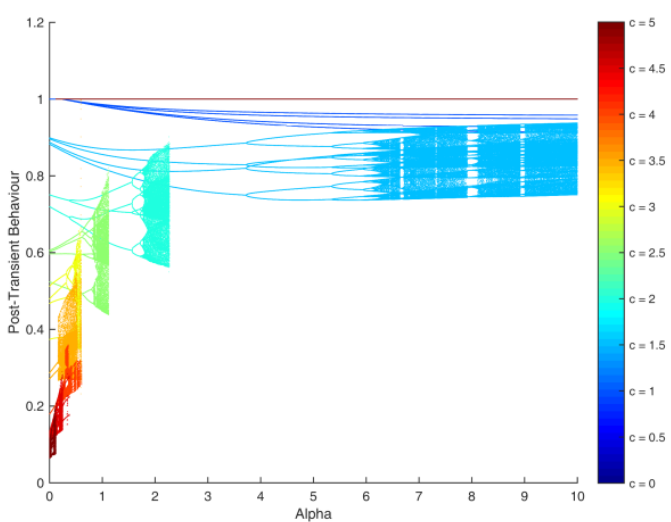

(b)

Figure 13. Bifurcation diagrams showing behaviour at fixed values of $c$ for varying $\alpha$ between 0 and 10 with a fixed mutation rate of $p=0.49$ and the use of the Ricker map based predator-prey model, (a) shows the predator behaviour and (b) shows the $1^{\text {st }}$ prey species $\left(N_{l}\right.$, with $\left.r_{l}=0.3\right)$ behaviour. 